\title{
Stress resultant nonlinear constitutive model for cracked reinforced concrete panels
}

\author{
Miquel Huguet ${ }^{\mathrm{a}, \mathrm{b}, *}$, Silvano Erlicher ${ }^{\mathrm{a}}$, Panagiotis Kotronis ${ }^{\mathrm{b}}$, François Voldoire ${ }^{\mathrm{c}, \mathrm{d}}$ \\ ${ }^{a}$ EGIS Industries, 4 Dolorès Ibarruri, 93188 Montreuil, France \\ ${ }^{\mathrm{b}}$ Ecole Centrale de Nantes, Université de Nantes, CNRS, Institut de Recherche en Génie Civil et Mécanique (GeM), 1 rue de la Nöe, F-44321 Nantes, France \\ 'Électricité de France, RED/AMA, 7, bld. Gaspard Monge, F-91120 Palaiseau, France \\ ${ }^{\mathrm{d}}$ Institut des Sciences de la Mécanique et Applications Industrielles, UMR EDF-CNRS-CEA-ENSTA 9219, 7, bld. Gaspard Monge, F-91120 Palaiseau, France
}

The paper proposes a novel stress resultant nonlinear constitutive model for Reinforced Concrete (RC) panels adapted to cyclic loadings. An analytical multi-scale analysis is applied by taking a concrete strut with embedded steel reinforcement between two consecutive cracks as representative volume element. Some suitable assumptions are adopted in order to incorporate the most important nonlinear phenomena characterizing reinforced concrete behavior: concrete damage, concrete cracking, bond-slip stress (at the origin of the tension stiffening effect) and steel yielding. The model is validated by comparison with experimental data concerning tension and tension-compression uniaxial tests on RC beams and a cyclic (non-reversing) shear test on an RC wall.

\section{Introduction}

\subsection{Nonlinear finite element modeling of reinforced concrete panels}

Industrial buildings, in particular Nuclear Power Plants (NPP), have to fulfill severe structural requirements according to the modern design codes. The computational time required for nonlinear structural analyses of this type of large-dimension Reinforced Concrete (RC) facilities, sometimes necessary for their seismic assessment, is significant. However, the so-called global or effective modeling approaches can ensure numerical efficiency and robustness. These approaches are characterized by the use of relatively large size Finite Elements (FE) where the material model represents the reinforced concrete behavior as an equivalent homogeneous material, as opposed to approaches based on distinct concrete and steel modeling and the introduction of some kinematic and/or stress transfer conditions. In civil engineering, this type of global modeling strategy is usually coupled with linear elastic behavior assumptions.

Nevertheless, recent safety requirements for NPP have introduced the necessity of using more realistic models able to reproduce the actual nonlinear behavior of RC structures, both for static and dynamic load cases. In particular, these models should be able to take into account the cracking onset and its development, in order to correctly estimate the crack widths (and also spacing and direction) since engineering design standards provide some bounds to these values to fulfill prescribed

\footnotetext{
* Corresponding author at: EGIS Industries, 4 Dolorès Ibarruri, 93188 Montreuil, France.

E-mail addresses: miquel.huguet-aguilera@egis.fr (M. Huguet), silvano.erlicher@egis.fr (S. Erlicher), panagiotis.kotronis@ec-nantes.fr (P. Kotronis), francois.voldoire@edf.fr (F. Voldoire).
} 


\section{Nomenclature}

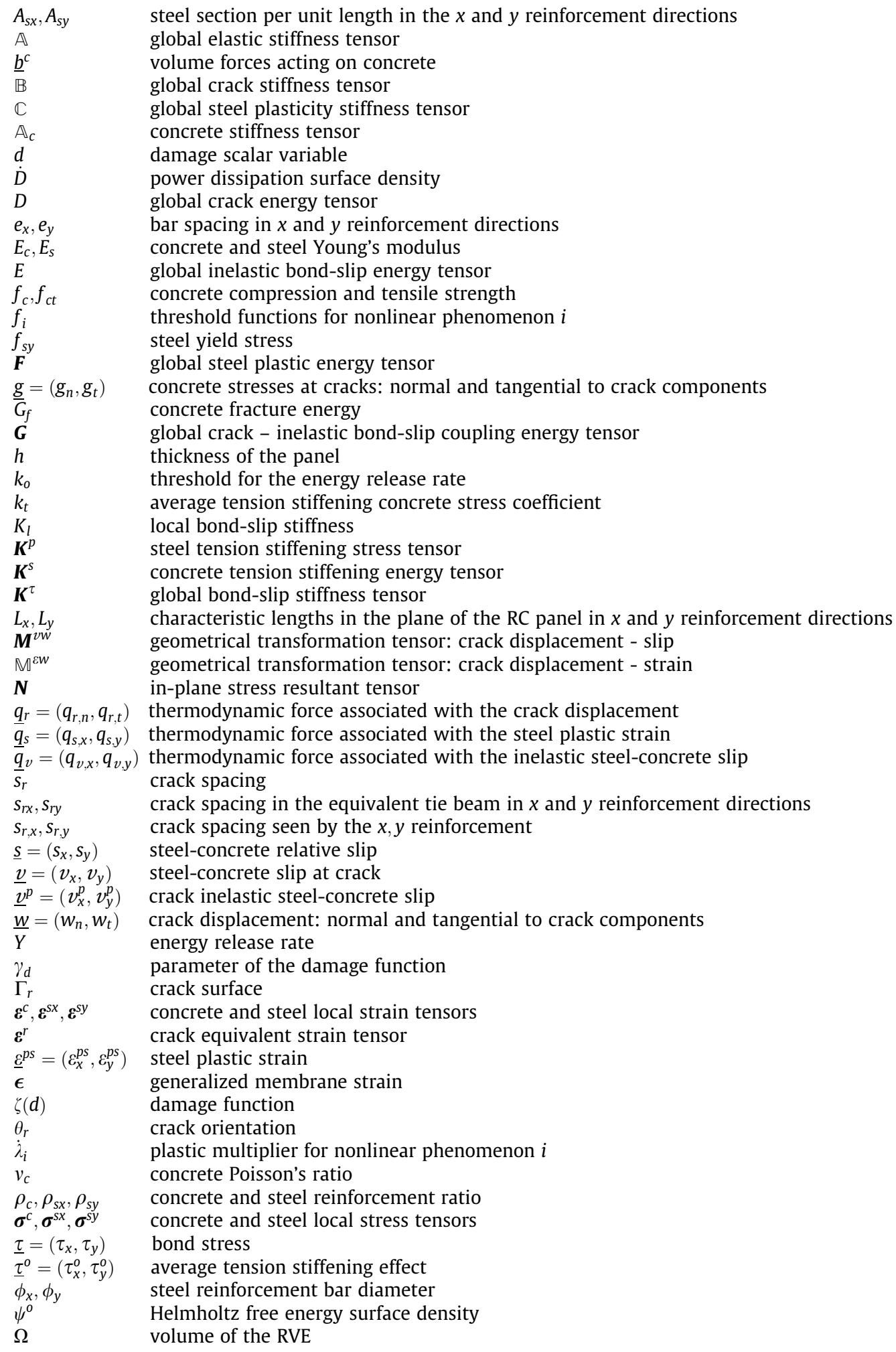




\author{
Sub/superscripts \\ $i=d, g n, g t, v x, v y, s x, s y$ nonlinear phenomena \\ $r$ \\ $\alpha=x, y \quad$ each of the two reinforcement orthogonal directions \\ $\omega=c, s x, s y \quad$ each of the materials of reinforced concrete: Concrete and steel reinforcement in $x$ and $y$ directions
}

aesthetic, durability and confinement (for NPP) conditions, or to ensure the strength of equipment anchorage. Concrete stiffness reduction (as a critical contribution to the dynamic structural behavior), steel yielding and permanent strains are other important parameters in structural design which should be accurately assessed.

Concerning RC panels, a relatively important number of global nonlinear models able to reproduce the development of cracks have been recently developed. They can be split into two categories.

On the one hand, the so-called phenomenological approaches describe cracking in RC panels adopting suitable assumptions or laws for all the physical phenomena governing the nonlinear structural response. Since they are developed and calibrated from an important number of experimental tests, they are the result of a deep understanding of the behavior of RC structures. Nevertheless, these approaches often lead to models that are only applicable for design cases similar to the experimental campaigns adopted for the calibration, usually only under monotonic loading conditions. Furthermore, their numerical implementation requires computational expensive iterations to satisfy both equilibrium and constitutive modeling conditions at the local scale and the link with the global scale is not explicitly described. The following constitutive models belong to this class. The Modified Compression Field Theory (MCFT) of Vecchio and Collins [30] is a fully rotating, smeared, stress-free crack model where equilibrium, compatibility and stress-strain relations are formulated in terms of average strains and stresses; an orthotropic concrete model with compression softening and tension stiffening effects is adopted. The Disturbed Stress Field Model (DSFM) of Vecchio [29] is an extension of the previous model allowing the principal stress and strain directions to be different by considering the tangential slip at cracks and the transmitted shear stress (aggregate interlock). The Cracked Membrane Model (CMM) developed by Kaufmann [16] and Kaufmann and Marti [17] and the Extended Cracked Membrane Model (ECMM) developed by Pimentel et al. [22] can be seen respectively as equivalent of MCFT and DSFM, respectively, when establishing equilibrium equations directly at cracks, since they both take into account concrete compression softening and tension stiffening, the former being a rotating crack approach and the latter a fixed crack one enabling stress transfer through cracks. The Softened Membrane Model (SMM) of Hsu and Zhu [15] applies the principles of Continuum Mechanics to the orthotropic continuous cracked (smeared) RC material. Finally, the PARC model developed by Belletti et al. [1] is developed from equilibrium of an RC strut between two consecutive cracks and accounts for dowel action, aggregate interlock, bridging effect, tension stiffening, and concrete softening in compression.

On the other hand, several constitutive models based on the global (or stress-resultant) modeling approach are directly formulated for an FE implementation at the structural element scale: the in-plane stress resultant $\boldsymbol{N}$ is explicitly expressed as a function of the generalized membrane strains $\epsilon$ and $n$ internal variables $\underline{\alpha}=\left(\alpha_{1}, \ldots, \alpha_{n}\right)$ whose role is to reproduce the nonlinear cyclic response of RC panels. The corresponding evolution laws are formulated within the framework of the Thermodynamics of Irreversible Processes (TIP) (see e.g. [9,10,18,23]), where the state of the material is defined by the Helmholtz free energy surface density $\psi^{\circ}(\boldsymbol{\epsilon}, \underline{\alpha})$, whose gradient define the non-dissipative thermodynamic forces (index $n d$ ) by the state equations:

$$
\boldsymbol{N}=\boldsymbol{N}^{n d}:=\frac{\partial \psi^{0}(\boldsymbol{\epsilon}, \underline{\alpha})}{\partial \boldsymbol{\epsilon}} \quad-q_{i}^{d}=q_{i}^{n d}:=\frac{\partial \psi^{0}(\boldsymbol{\epsilon}, \underline{\alpha})}{\partial \alpha_{i}}
$$

The index $d$ (hereinafter omitted) stands for dissipative thermodynamic forces (hereinafter thermodynamic forces).

By only considering isothermal transformations, the second principle of TIP, which ensures the condition of a nonnegative power dissipation surface density $\dot{D}$, can be expressed as:

$$
\dot{D}=\boldsymbol{N}: \dot{\boldsymbol{\epsilon}}-\dot{\psi}^{o}(\boldsymbol{\epsilon}, \underline{\alpha})=\boldsymbol{N}: \dot{\boldsymbol{\epsilon}}-\frac{\partial \psi^{0}}{\partial \boldsymbol{\epsilon}}: \dot{\boldsymbol{\epsilon}}-\sum_{i} \frac{\partial \psi^{0}}{\partial \alpha_{i}} \cdot \dot{\alpha}_{i}=\sum_{i} q_{i} \cdot \dot{\alpha}_{i} \geqslant 0
$$

Moreover, in the considered models, the evolution of the internal variables is defined according to the Generalized Standard Materials Theory (GSMT) (see [13]), which allows a well-defined energetic characterization and entails that the time integration algorithm is associated with a well-posed minimization problem. According to this theory, the internal variable evolution is given by the normality rule:

$$
\dot{\alpha}_{i}=\dot{\lambda}_{i} \frac{\partial f_{i}}{\partial q_{i}}
$$

where the threshold functions $f_{i}$ depend in general on $f_{i}\left(q_{i}, \boldsymbol{\epsilon}, \underline{\alpha} ; \mu\right)$, with $\mu$ a set of variables acting as parameters (without associated thermodynamic forces). Threshold functions $f_{i}$ are differentiable and convex with respect to $q_{i}$ for any $\epsilon, \underline{\alpha}, \underline{\mu}$ set. 
They define the elastic domain for each nonlinear mechanism by satisfying, together with their associated plastic multipliers $\dot{\lambda}_{i}$, the Kuhn-Tucker conditions:

$$
\dot{\lambda}_{i} \geqslant 0 \quad f_{i} \leqslant 0 \quad \dot{\lambda}_{i} f_{i}=0
$$

This type of model formulation ensures a high degree of robustness and versatility to any dynamic load case that can occur during an RC building FE analysis and has shown good performances when applied to plain concrete [24]. GLRC_DM [20] and DHRC [5,6] are two RC plate nonlinear constitutive models of this type implemented in the Code_Aster FE software [8]. The first one consists in a damage model while the latter couples irreversible strains (induced by steel rebar debonding) with concrete damage. Nevertheless, these constitutive models are not always able to reproduce accurately the nonlinear behavior for RC plates under severe load cases. In particular, their range of validity is limited to a moderate nonlinear response of RC plates, in the Serviceability Limit State domain, as defined in Eurocode2 (EC2) [3]. Therefore, they cannot give accurate results concerning the actual local cracking state of RC elements and the yielding of the steel reinforcement bars.

Moreover, it is noticed that GLRC_DM and DHRC models have been built using two different multi-scale analyses: a heuristic homogenization process for the former and a fully justified numerical averaging method applied on the mechanical fields on a Representative Volume Element (RVE) for the latter. The multi-scale analysis, often applied in civil engineering to derive equivalent RC plate constitutive relations, has been introduced in the literature for many decades and applied in different engineering fields. For example, the homogenization technique has been justified using an asymptotic expansion method on three-dimensional elasticity equations, leading to the well-known bi-dimensional linear plate theory (see [4]), assuming that both underlying small parameters (the ratio of the thickness over the plate dimensions, and the ratio of the heterogeneities size over the plate thickness) are of the same order. A first attempt to apply this method to an RC plate, limited to the linear elastic range, was proposed by Destuynder and Theodory [7]. The authors established the membrane and flexural equivalent stiffness tensors in terms of stress resultant in the RC plate, after solving the underlying linear elastic auxiliary problems on a periodic unit cell, or RVE, including both concrete and steel grids, and average value calculations.

\subsection{New stress resultant nonlinear model for RC panels}

The aim of this paper is to propose a novel stress resultant (global) constitutive model for RC panels suitable for a robust FE implementation and able to reproduce the nonlinear response under cyclic solicitations in the entire SLS domain. The onset of steel yielding, which usually characterizes the beginning of the Ultimate Limit State (ULS) of RC elements, is also modeled.

The previously mentioned phenomenological models are the result of a deep understanding of the behavior of RC. Therefore, they are a consistent physical basis for the development of the new model and their local scale descriptions focusing on local displacement and stress variables are adopted. Inspired by these models, the following four physical nonlinear phenomena are considered:

- Isotropic concrete damage in compression,

- Concrete cracking, considering both normal and tangential-to-the-crack displacements and stress transfer,

- Bond stress at the steel-concrete interface caused by their relative slip and causing the tension stiffening effect (e.g. [21]),

- Yielding of steel reinforcement bars occurring locally at cracks, where the maximum (in absolute value) steel stresses are reached.

The link between these local phenomena on the one hand and the global scale model on the other hand is made by means of a closed-form multi-scale analysis, adopting suitable assumptions. The principles of this multi-scale analysis applied on a cracked RC panel are presented in Section 2. The local scale description of the four nonlinear phenomena taken into account by the model is presented in Section 3.

With the previous elements, a closed-form multi-scale analysis is applied on an RC panel in the stabilized crack state configuration and is presented in Section 4. Then, Section 5 is devoted to the formulation of the general form of the obtained stress-resultant constitutive model in the well-defined theoretical framework of TIP and GSMT, which guarantees an efficient FE software implementation, adapted to all types of load paths (including cyclic ones).

Finally, in Section 6, the new model is applied to uniaxial pure tension and tension-compression tests on RC beams and to a global shear cyclic test (without inversion of the sign of the applied force) on a RC wall, in order to highlight its capacity to reproduce experimental results concerning both global (force-displacement) and local (crack widths) values.

\section{Preliminary considerations about the multi-scale approach}

The RC panel of Fig. 2-1 submitted to in-plane loads, without flexural effects, is considered. The $x$ and $y$ axes define the membrane plane and the direction of the two orthogonal groups of steel reinforcement bars, characterized by their diameters $\phi_{x}$ and $\phi_{y}$ and spacing $e_{x}$ and $e_{y}$, respectively. The geometry of the panel is defined by the in-plane dimensions $L_{x}$ and $L_{y}$ and its thickness $h$. The three different components of the RC panel are identified with $\omega=c, s x, s y$ for concrete, $x$ steel bars and $y$ steel bars respectively. 


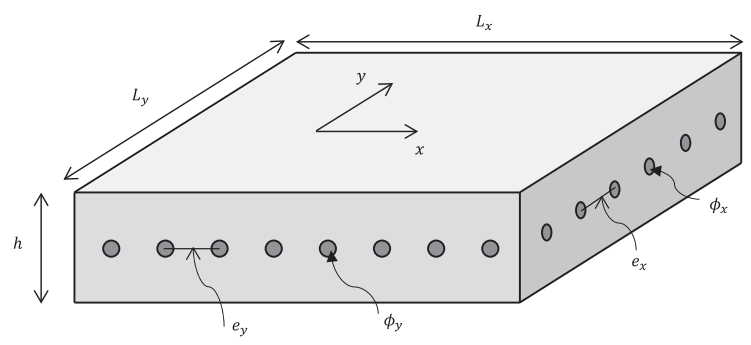

Fig. 2-1. Geometry of the RC panel.

\subsection{Overview of the proposed analytical multi-scale analysis}

In the RC panel of Fig. 2-1, two different scales can be identified:

- The local scale (or micro-scale) is the level of the three material components (concrete, $x$ steel bars and $y$ steel bars).

- The global scale (or macro-scale) is the level of the resultant stress $\boldsymbol{N}$, the generalized strain $\boldsymbol{\epsilon}$ and other global state variables describing reinforced concrete as an equivalent homogeneous material. The FE discretization is done at this scale.

The multi-scale analysis allows obtaining the global scale relationship between the stress resultant and the generalized strain as a result of the laws describing the physical phenomena occurring at the local scale. Local mechanisms are analyzed within the RVE of the problem, which is the smallest volume able to represent the physical phenomena governing the global response of the material (the RVE dimensions are smaller than the spatial distribution length of the nonlinear mechanisms) and which is assumed to be periodically repeated in space. Therefore, the suitability of the chosen RVE is essential to obtain representative results at the global scale.

After the choice of a suitable RVE of the problem, the procedure of Fig. 2-2 is applied in order to obtain the global scale relationship between the stress resultant and the generalized strain. Thus, the proposed multi-scale analysis consists of the following 5 steps:

(1) Definition of the RVE and its main geometrical and mechanical properties, in Section 4.1.

(2) Definition of the local stress fields $\boldsymbol{\sigma}^{(\omega)}=\boldsymbol{\sigma}^{c}, \boldsymbol{\sigma}^{s x}, \boldsymbol{\sigma}^{s y}$ (stresses in concrete, $x$ steel bars and $y$ steel bars respectively) as functions of the applied stress resultant $\boldsymbol{N}$ (stress localization), in Section 4.2.

(3) Application of the local constitutive laws to obtain the local strain fields $\boldsymbol{\varepsilon}^{\omega}=\boldsymbol{\varepsilon}^{c}, \boldsymbol{\varepsilon}^{s x}, \boldsymbol{\varepsilon}^{s y}$ (strains in concrete, $x$ steel bars and $y$ steel bars respectively), in Section 4.3.

(4) Application of the compatibility equations and the averaging method to obtain the generalized strain field $\epsilon$, in Section 4.4 .

(5) Resolution of the obtained equations in order to establish the $\boldsymbol{N}-\boldsymbol{\epsilon}$ relationship and other results of interest at the global scale, in Section 4.5 .

Differently from the homogenization procedure implemented to define the DHRC model, where prior FE computations are carried out to identify the parameters of the global constitutive model before the RC structure FE analysis, it has been decided to directly address the stress field in order to include the local equilibrium equations in the local state description. Therefore, a closed-form multi-scale analysis is retained and performed in Section 4 after assuming a suitable hypotheses set.

\section{Global constitutive law}

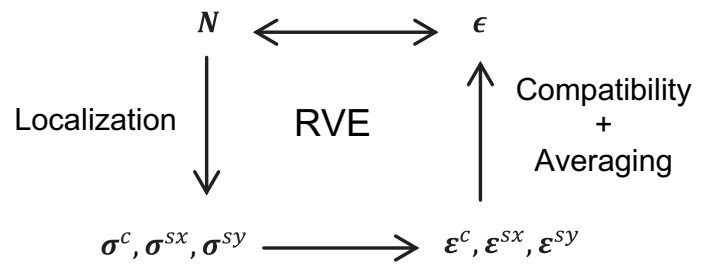

Local constitutive laws

Fig. 2-2. Diagram of the multi-scale analysis. 


\subsection{Averaging method}

The averaging method, which is a technique to link global and local fields, is based on the definition of the average of a given local field defined in a volume $\Omega$ as:

$$
\langle\cdot\rangle_{\Omega}=\frac{1}{|\Omega|} \int_{\Omega} \cdot d v
$$

This definition is used to calculate the membrane generalized strain tensor $\epsilon$ from the average values of the planar components of the local strain tensor for a displacement field $\underline{u}$ as follows:

$$
\boldsymbol{\epsilon}=\langle\boldsymbol{\varepsilon}(\underline{u})\rangle_{\Omega}=\frac{1}{|\Omega|} \int_{\Omega} \boldsymbol{\varepsilon}(\underline{u}) d v
$$

Considering the possibility of a displacement discontinuity $\llbracket u \rrbracket$ on a particular piece-wise regular boundary $\Gamma$ in the RVE typically a crack - (denoting by $\underline{n}$ the local unit outer normal vector on $\Gamma$ and by $\otimes^{s}$ the symmetric dyadic tensor product), the following extension of (2-2) is made (by application of the Stokes' theorem):

$$
\boldsymbol{\epsilon}=\langle\boldsymbol{\varepsilon}(\underline{u})\rangle_{\Omega}=\frac{1}{|\Omega|}\left(\int_{\Omega} \boldsymbol{\varepsilon}(\underline{u}) d v+\int_{\Gamma} \llbracket \underline{u} \rrbracket \rrbracket \otimes^{s} \underline{n} d S\right)
$$

Since the RC panel consists of concrete and steel bars in the $x$ and the $y$ directions, the total RVE domain $\Omega$ is the union of the three RC components independent subdomains $\Omega_{c}, \Omega_{s x}$ and $\Omega_{s y}$, so that the total volume is the sum of the three subdomains volumes:

$$
|\Omega|=\left|\Omega_{c}\right|+\left|\Omega_{s x}\right|+\left|\Omega_{s y}\right|
$$

Therefore, the stress-resultant tensor $\boldsymbol{N}$, defined as the resultant on the thickness $h$ of the average stress on the RVE, can be computed from local stress tensors (application of the macrohomogeneity static equilibrium principle):

$$
\boldsymbol{N}=h\langle\boldsymbol{\sigma}\rangle_{\Omega}=\frac{h}{|\boldsymbol{\Omega}|} \int_{\Omega} \boldsymbol{\sigma} d v=\frac{h}{|\boldsymbol{\Omega}|} \sum_{\omega} \int_{\Omega_{\omega}} \boldsymbol{\sigma}^{\omega} d v
$$

The local stress field average is defined in each subdomain $\Omega_{\omega}$ as:

$$
\langle\boldsymbol{\sigma}\rangle^{\omega} \equiv\left\langle\boldsymbol{\sigma}^{\omega}\right\rangle_{\Omega_{\omega}}=\frac{1}{\left|\Omega_{\omega}\right|} \int_{\Omega_{\omega}} \boldsymbol{\sigma}^{\omega} d v
$$

Hence, Eq. (2-5) can be expressed as:

$$
\boldsymbol{N}=h \sum_{\omega} \rho_{\omega}\left\langle\boldsymbol{\sigma}^{\omega}\right\rangle
$$

where the volume fraction of each material component $\omega=c, s x, s y$ is defined as $\rho_{\omega}=\left|\Omega_{\omega}\right| /|\Omega|$, which satisfy $\rho_{c}+\rho_{s x}+\rho_{s y}=1$ using (2-4).

Considering the RC panel of Fig. 2-1, the steel volume fraction is equal to the classical reinforcement ratio used in engineering codes, defined as:

$$
\rho_{s x}=\frac{\pi \phi_{x}^{2}}{4 h e_{x}} \quad \rho_{s y}=\frac{\pi \phi_{y}^{2}}{4 h e_{y}}
$$

\section{Material modeling at the local scale}

The application on a cracked RC panel of the general procedure for the multi-scale analysis presented in Section 2.1 depends on the assumptions on the local material behavior. In particular, in this section the four nonlinear physical phenomena considered in the model formulation are analyzed in order to have all the necessary elements to define the RVE of the problem and to apply the multi-scale procedure steps of Fig. 2-2.

\subsection{Concrete damage}

Concrete damage is caused by the onset and development of homogeneous diffuse micro-cracking. It results in a concrete stiffness reduction which is considered hereinafter as isotropic. Therefore, it is introduced as an internal damage variable $d$, scalar, positive and increasing. As usual in Damage Mechanics, this variable directly affects the concrete Young's modulus $E_{c}$ [18], by means of a decreasing convex function $\zeta(d)$, hence inducing an isotropic stiffness degradation. The relationship between the concrete membrane stress $\boldsymbol{\sigma}^{c}$ and the plane components of the strain tensor $\boldsymbol{\varepsilon}^{c}$ is given by: 


$$
\left(\begin{array}{l}
\sigma_{x x}^{c} \\
\sigma_{y y}^{c} \\
\sigma_{x y}^{c}
\end{array}\right)=\frac{E_{c} \zeta(d)}{1-v_{c}^{2}}\left(\begin{array}{ccc}
1 & v_{c} & 0 \\
v_{c} & 1 & 0 \\
0 & 0 & \frac{1-v_{c}}{2}
\end{array}\right) \cdot\left(\begin{array}{c}
\varepsilon_{x x}^{c} \\
\varepsilon_{y y}^{c} \\
\gamma_{x y}^{c}
\end{array}\right)
$$

Therefore, the concrete constitutive law is characterized by a stiffness tensor $\mathbb{C}_{c}(d)$ defined by the Young's modulus $E_{c}$, the Poisson coefficient $v_{c}$ and the decreasing convex function $\zeta(d)$, which in this work is retained with the same expression as the damage functions of GLRC_DM and DHRC models:

$$
\zeta(d)=\frac{1+\gamma_{d} d}{1+d}
$$

This damage function produces a bilinear strain-stress response for uniaxial monotonic loading paths when it is associated to a constant threshold for the energy release rate, see Combescure et al. [5]. The parameter $\gamma_{d}$ defines the tangent slope of the monotonic loading stress-strain curve (as a ratio with respect to the initial one) in the damage evolution phase, as well as the secant slope when damage tends to infinite.

\subsection{Concrete cracking}

Concrete cracking (onset and development of macro-cracks) is seen as localized concrete displacement discontinuities at an assumed sharp and plane crack surface $\Gamma_{r}$. It consists in a normal-to-crack component $w_{n}$ (crack width) and in a tangential-to-crack component $w_{t}$ (see Fig. 3-1), regrouped in the crack displacement variable $\underline{w}=\left(w_{n}, w_{t}\right)$. Similarly, it can be defined the vector of the concrete stress at cracks $\underline{g}=\left(g_{n}, g_{t}\right)$, which has also a normal $g_{n}$ and a tangential $g_{t}$ components with respect to the cracks.

Cracking onset occurs when the maximum principal concrete stress $\sigma_{1}^{c}$ reaches the concrete tensile strength $f_{c t}$. In the model, the orientation $\theta_{r}$ of the crack $\left(-\pi / 2<\theta_{r} \leqslant \pi / 2\right)$ is defined as the perpendicular direction to $\sigma_{1}^{c}$ at the cracking onset. In other words, the classical Rankine criterion is adopted:

$$
F_{c r}\left(\boldsymbol{\sigma}^{c}\right)=\sigma_{1}^{c}-f_{c t} \leqslant 0
$$

When cracking is developed following a $\theta_{r}$ orientation, the crack spacing (or distance between two consecutive cracks) can be calculated with the expression given by Vecchio and Collins [30]:

$$
s_{r}=\left(\frac{\left|\sin \theta_{r}\right|}{s_{r x}}+\frac{\cos \theta_{r}}{s_{r y}}\right)^{-1}
$$

where $s_{r x}$ and $s_{r y}$ are the theoretical average crack spacings of the equivalent tie beams in the $x$ and $y$ directions, respectively, and which can be calculated for example with the expressions given in Model Code 2010 (MC10) [12], EC2 or Huguet [14].

In an RC panel, cracks do not appear everywhere at the same time. As an example, regarding the cracking development in the RC wall number 3 subjected to a fixed direction incremental loading of the CEOS.fr French national project [25], three different cracking states can be distinguished:

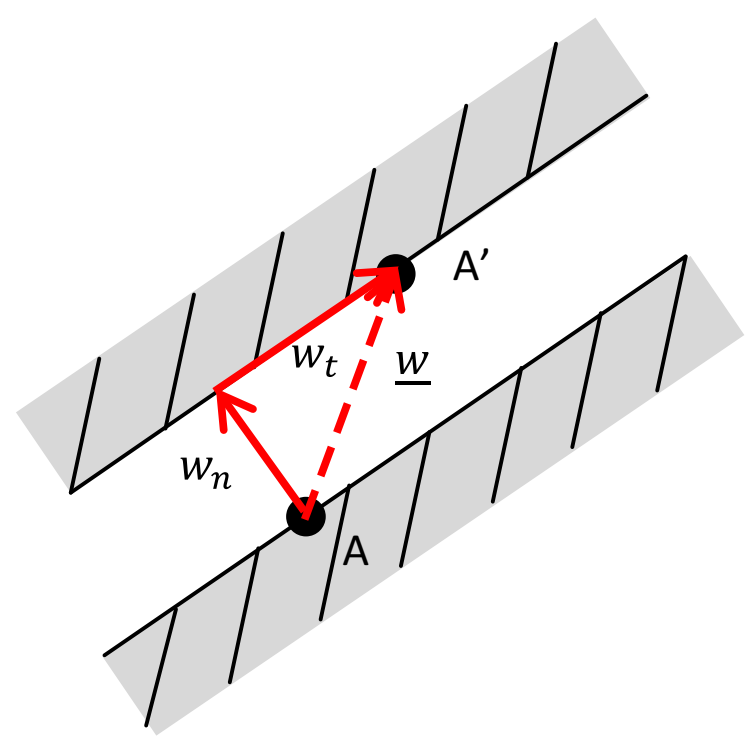

Fig. 3-1. Concrete displacement discontinuity $\underline{w}=\left(w_{n}, w_{t}\right)$ at a crack. 
(i) The uncracked state, where the relatively weak solicitations do not cause any crack.

(ii) The crack formation state, where one or more cracks exist in the RC panel and their number increases with increasing loading. The chronological apparition and distribution of cracks are governed by the non-uniform state of stress, the concrete properties variability (e.g. tensile strength variability) and the influence of the existing cracks. As an example, the onset of the first crack in the CEOS.fr RC wall number is shown in Fig. 3-2(a).

(iii) The stabilized cracking state, characterized by a fixed crack pattern that does not change even with increasing loading, even if the crack displacement $\underline{w}$ evolves. Obviously, this is a somehow idealised state since more cracks may appear for very important loadings. As an example, the crack pattern of Fig. 3-2(b) can be considered as stabilized (at least in its right half) as long as no new cracks appear when the load is augmented by $17 \%$, see Fig. 3-2(c).

These cracking states are also identified and described in MC10. It should be mentioned that a change in the loading direction causes a new stress field that may form new cracks in different directions, even when the stabilized crack state has been reached. However, for the case of RC panels, when a fixed crack pattern is established a new solicitation does not cause new cracks unless the new loading direction is different enough from the initial one. This experimental evidence is at the origin of the fixed crack approaches (e.g. ECMM), as opposed to the rotating ones that update the orientation of active cracks at every loading step (e.g. MFCT and CMM).

The typical plate finite elements used to model an RC wall may contain few cracks within it. Therefore, the crack formation state within a finite element can be considered limited in time since the first and last crack onsets occur very closely. Thus, only two states are taken into account in the formulation of the present model, clearly distinguished by the cracking criterion (3-3): the uncracked and the stabilized states. Consequently, in Section 4 the multi-scale analysis is performed in the framework of a stabilized crack pattern and in Section 5 the obtained results are generalized to take also into account the uncracked phase.

\subsection{Steel-concrete bond-slip}

Bond stresses $\underline{\tau}=\left(\tau_{x}, \tau_{y}\right)$ are transmitted from the $x$ and $y$ reinforcement steel bars to concrete. They occur when a relative slip $\underline{s}=\left(s_{x}, s_{y}\right)$ between these materials appears. In particular, in this work the assumption of a linear local bond-slip law with a bond-slip stiffness $K_{l}$ in the $\alpha=x, y$ direction is retained:

$$
\tau_{\alpha}=K_{l} s_{\alpha}
$$

Both $\underline{\tau}$ stresses and $\underline{s}$ slip vary along the steel reinforcement bars between two consecutive cracks. At the global scale, the only visible and measurable concrete-steel relative displacement is the slip at cracks $\underline{v}=\left(v_{x}, v_{y}\right)$, which can be geometrically related to the crack displacement $\underline{w}$ by means of the geometrical matrix $\boldsymbol{M}^{v w}\left(\theta_{r}\right)$ :

$$
\underline{v}=\boldsymbol{M}^{v w}\left(\theta_{r}\right) \cdot \underline{w}=\left(\begin{array}{cc}
\sin \left|\theta_{r}\right| & -\cos \theta_{r} \operatorname{sign}\left(\theta_{r}\right) \\
\cos \theta_{r} & \sin \theta_{r}
\end{array}\right) \cdot \underline{w}
$$

(a)

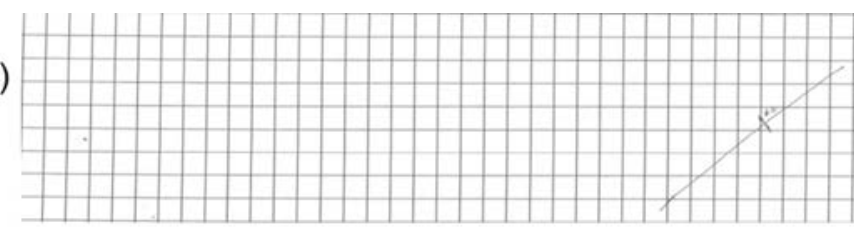

(b)

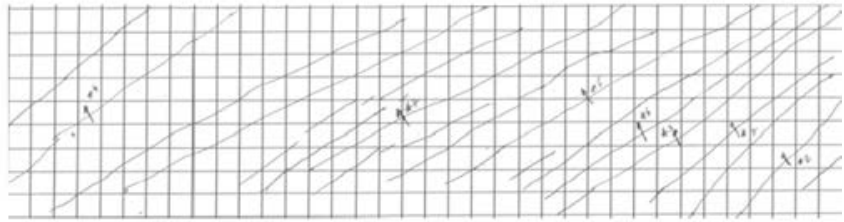

(c)

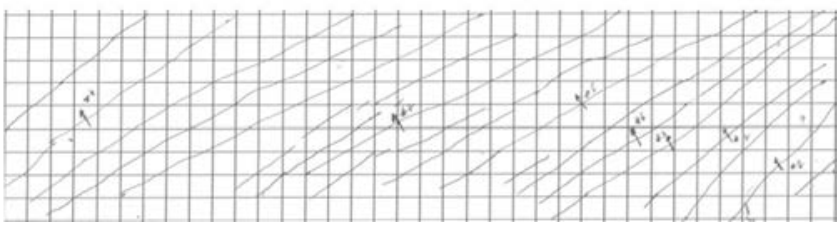

Fig. 3-2. Crack pattern in the RC wall number 3 of CEOS.fr project [25] at (a) $900 \mathrm{KN}$, (b) $3600 \mathrm{KN}$ and (c) $4200 \mathrm{KN}$ load levels. 
Moreover, in the formulation of the global constitutive model the bond slip stress appears under the form of an average tension stiffening effect $\underline{\tau}^{0}$ (4-20) which, as shown in (A-5) of Appendix A, can also be expressed as a function of the slip at cracks $\underline{v}$ by means of the global bond-slip stiffness tensor $\boldsymbol{K}^{\tau}$ defined in (4-23):

$$
\underline{\tau}^{0}=\boldsymbol{K}^{\tau} \cdot \underline{v}=\boldsymbol{K}^{\tau} \cdot \boldsymbol{M}^{v w}\left(\theta_{r}\right) \cdot \underline{w}
$$

Finally, the inelastic steel-concrete slip variable $\underline{v}^{p}=\left(v_{x}^{p}, v_{y}^{p}\right)$ is introduced, the inelastic part of the slip between the concrete and the steel reinforcement bars at cracks that allows the formulation of a global nonlinear law for the bond slip stress by generalizing (3-7):

$$
\underline{\tau}^{0}=\boldsymbol{K}^{\tau} \cdot\left(\underline{v}-\underline{v}^{p}\right)=\boldsymbol{K}^{\tau} \cdot\left(\boldsymbol{M}^{v w}\left(\theta_{r}\right) \cdot \underline{w}-\underline{v}^{p}\right)
$$

\subsection{Yielding of steel reinforcement bars}

The steel reinforcement bars are supposed to be a one-dimensional medium and to carry only longitudinal forces. Moreover, their behavior is modeled using an elastic - plastic constitutive law:

$$
\boldsymbol{\sigma}^{s \alpha}=E_{s}\left(\varepsilon_{\alpha \alpha}^{s \alpha}-\varepsilon_{\alpha}^{p s}\right) \underline{e}_{\alpha} \otimes \underline{e}_{\alpha}
$$

where $\otimes$ denotes the tensor dyadic product, $\boldsymbol{\sigma}^{s \alpha}$ is the membrane stress tensor, $E_{s}$ is the steel Young's modulus, and $\varepsilon_{\alpha \alpha}^{s \alpha}$ and $\varepsilon_{\alpha}^{p s}$ are the total and plastic longitudinal strains respectively, for the steel bars in the $\alpha=x, y$ direction. However, since in this model the yielding of steel reinforcement bars is assumed to be located and concentrated at the crack crossings (see Section 5.3.4), the vector variable steel plastic strain $\underline{\varepsilon}^{p s}=\left(\varepsilon_{x}^{p s}, \varepsilon_{y}^{p s}\right)$ is introduced, which regroups the plastic strains at cracks of $x$ and $y$ steel bars.

\subsection{Summary of the retained state variables}

In the framework of TIP, the variables describing the state of the material (state variables) are classified in observable variables, accounting for the reversible part of the material behavior, and internal variables, which define the irreversible and history-dependent effects.

The local observable variables identified in this section are the concrete and steel strain tensors $\varepsilon_{\alpha \beta}^{c}$ and $\varepsilon_{\alpha \alpha}^{s \alpha}$ respectively. The retained internal variables are associated to each of the four considered nonlinear physical phenomena:

- Damage variable $d$, accounting for the irreversibility of concrete stiffness reduction,

- Crack displacement $\underline{w}=\left(w_{n}, w_{t}\right)$, associated with concrete cracking,

- Inelastic steel-concrete slip $\underline{v}^{p}=\left(v_{x}^{p}, v_{y}^{p}\right)$,

- Steel plastic strain at crack crossings $\underline{\varepsilon}^{p s}=\left(\varepsilon_{x}^{p s}, \varepsilon_{y}^{p s}\right)$.

\section{Analytical multi-scale analysis of a cracked RC panel}

As concluded in Section 3.2, the analytical multi-scale analysis (defined by the steps of Section 2.1) is applied to an RC panel in the stabilized cracking state.

The crack pattern of the RC panel of Fig. 4-1(a) is characterized by the average crack orientation $\theta_{r}\left(-\pi / 2<\theta_{r} \leqslant \pi / 2\right)$ and the average crack spacing. Since a global approach is considered for the formulation of the model, the crack-to-crack (and also along one single crack) variation of these parameters is not taken into account when using their average values. The crack orientation defines the in-plane $t-n$ local axes (tangent and normal to the crack) while $z$ is the out-of-plane axis. The relationship between overall $(x-y)$ and local $(t-n)$ axes for any vector $\underline{u}$ is given by:

$$
\left(\begin{array}{l}
u_{x} \\
u_{y}
\end{array}\right)=\left(\begin{array}{cc}
\cos \theta_{r} & -\sin \theta_{r} \\
\sin \theta_{r} & \cos \theta_{r}
\end{array}\right) \cdot\left(\begin{array}{l}
u_{t} \\
u_{n}
\end{array}\right)
$$

\subsection{The RVE of the cracked RC panel}

In the stabilized cracked configuration of Fig. 4-1(a), the panel is considered as a repetitive succession of straight RC struts of thickness $h$ (thickness of the panel), oriented in the $\theta_{r}$ direction and separated by two successive crack surfaces (crack spacing $s_{r}$ ), whatever the distance of two consecutive reinforcement steel bars. Therefore, inspired by the Ritter-Mörsch scheme, these struts are chosen as the RVE of the problem as presented in Fig. 4-1(b). In the following, it is supposed that the typical size of the finite elements used to model the RC panel is larger than the smallest dimension of the RVE, and so larger than the crack spacing. Moreover, the following assumptions are adopted within the chosen RVE:

(i) The local stress fields $\boldsymbol{\sigma}^{\omega}$ are considered constant along the $t$ direction, because the boundary strains/stresses are constant on the entire crack boundary $\Gamma_{r}$ and the RVE dimension in the $n$ direction is the crack spacing $s_{r} \ll L_{x}, L_{y}$. 


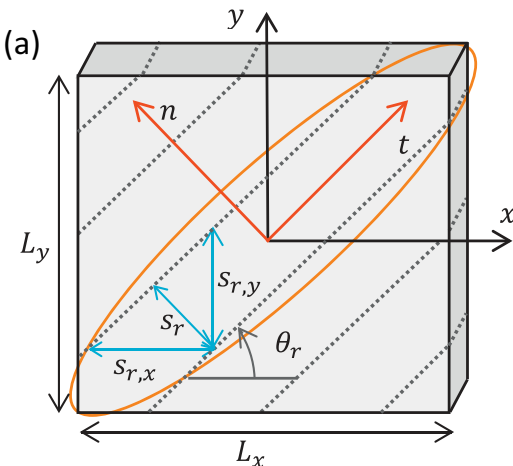

(b)

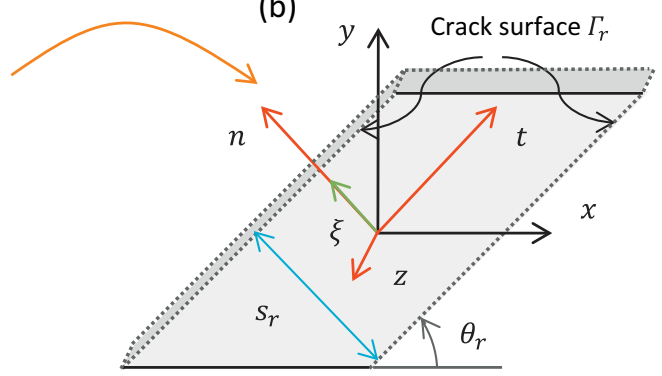

Fig. 4-1. Determination of the RVE from the idealization of a cracked RC panel and geometry definition.

(ii) All fields are considered constant in the $z$ direction within the panel thickness $h$. Although in RC structures a concentration of concrete stresses and strains often occurs around the steel bars, this phenomenon is not taken into account for the sake of simplicity.

(iii) The plane $t-z$ is a symmetry plane.

(iv) The reinforcement steel bars (but also the concrete) are considered uniformly smeared in the RVE, since $e_{x}, e_{y} \ll L_{x}, L_{y}$, see Fig. 2-1.

(v) The RVE is not submitted to external body forces (self-weight is considered only at the global scale).

Following the assumptions (i) and (ii), the fields only depend on the $\xi$ dimensionless coordinate along the $n$ axis, varying from -1 to +1 :

$$
\xi(x, y)=\frac{2}{s_{r}}\left(-x \sin \theta_{r}+y \cos \theta_{r}\right)
$$

and the average value (2-1) in the RVE is calculated according to:

$$
\langle\cdot\rangle_{\Omega}=\langle\cdot(\xi)\rangle_{\Omega}=\frac{1}{2} \int_{-1}^{1} \cdot(\xi) d \xi
$$

Following the assumption (iv), steel reinforcement bars and concrete are considered as continuous media into the entire RVE.

Moreover, only surface forces are applied at the frontier with the adjacent RVE and the $\boldsymbol{N}$ stresses are transmitted throughout these boundaries. According to the previous assumptions, Eq. (2-7) can be applied not only as an average but also at any point of the RVE:

$$
\boldsymbol{N}=h \sum_{\omega} \rho_{\omega} \boldsymbol{\sigma}^{\omega}(\xi) \quad \forall \xi \in[-1,1]
$$

Suquet [28] has shown that, in a homogenization technique, the microscopic internal variables have to be chosen (or proved to be) piecewise or discrete in the RVE in order to ensure a finite number of macroscopic internal variables when obtaining the resulting Generalized Standard macroscopic model.

It has been decided to keep this condition in the performed analytical multi-scale analysis. The internal variables crack displacement $\underline{w}$, inelastic steel-concrete slip $\underline{v}^{p}$ and steel plastic strain $\underline{\varepsilon}^{p s}$ are only defined at the RVE boundaries (the crack), so they do not vary in the RVE. However, the concrete damage variable $d$ may vary in the RVE in a general case, but in order to satisfy the previous condition of Suquet [28], the assumption of $d$ being constant in the entire RVE is adopted.

\subsection{Localization of the stress resultant}

In the retained RVE, concrete equilibrium at any point of $\Omega_{c}$ reads:

$$
\underline{\nabla} \cdot \boldsymbol{\sigma}^{c}(\xi)+\underline{b}^{c}(\xi)=\underline{0}
$$

where $\underline{\nabla}=(\partial / \partial x, \partial / \partial y)$ and the volume force vector $\underline{b}^{c}(\xi)=\left(b_{x}^{c}(\xi), b_{y}^{c}(\xi)\right)$. In the $\alpha$ direction, the volume forces are only caused by the smeared steel bars in the same direction, see assumptions (i) and (iv) of Section 4.1. They are defined as the product of the $n_{\alpha}$ bars contained in an infinitesimal concrete volume $\rho_{c} d v$ (with $d v=|d \Omega|=d x \cdot d y \cdot h$ ) by the infinitesimal force $d F_{\alpha}(\xi)$ exerted from each of these bars, divided by the infinitesimal concrete volume: 


$$
b_{\alpha}^{c}(\xi)=\frac{n_{\alpha} d F_{\alpha}(\xi)}{\rho_{c} d v}
$$

The differential force is the product of the tangential steel-concrete interface stress $\tau_{\alpha}(\xi)$ by the bar perimeter and by the differential length $d \alpha$ in the $\alpha$ direction:

$$
d F_{\alpha}(\xi)=\tau_{\alpha}(\xi) \pi \phi_{\alpha} d \alpha
$$

The steel ratio in the $\alpha$ direction (2-8) can be rewritten as:

$$
\rho_{s \alpha}=\frac{\left|d \Omega_{s \alpha}\right|}{|d \Omega|}=\frac{n_{\alpha} \pi \frac{\phi_{\alpha}^{2}}{4} d \alpha}{d v} \rightarrow n_{\alpha}=\frac{\rho_{s \alpha} d v}{\pi \frac{\phi_{\alpha}^{2}}{4} d \alpha}
$$

Substituting (4-7) and (4-8) in (4-6), the volume forces acting in concrete due to the interaction with the steel reinforcement bars by means of the bond stress $\tau_{\alpha}(\xi)$ are obtained:

$$
b_{\alpha}^{c}(\xi)=\frac{4 \rho_{s \alpha}}{\phi_{\alpha} \rho_{c}} \tau_{\alpha}(\xi)
$$

Using (4-2) and (4-9), the system of equilibrium Eq. (4-5) becomes:

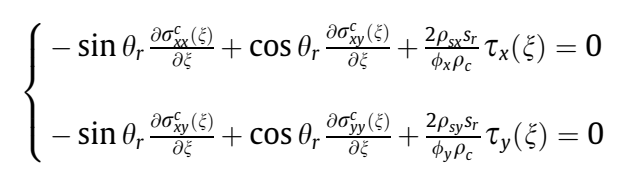

The boundary conditions for the concrete stress field are given by the stresses at cracks:

$$
\left\{\begin{array}{l}
\sigma_{n n}^{c}(\xi= \pm 1)=g_{n} \\
\sigma_{t n}^{c}(\xi= \pm 1)=g_{t}
\end{array}\right.
$$

The nine equations system necessary to determine the nine unknowns of the localization problem (the three plane-stress tensor components for each of the three components of the RVE) is obtained using the two concrete equilibrium equations (4-10), the three equations resulting for the stress averaging adapted to the geometry of the RVE (4-4), and the four equations coming from the constitutive hypothesis of steel bars, which are supposed to carry stresses only in their longitudinal direction, see (3-9):

$$
\left\{\begin{array}{l}
\frac{\nabla}{\boldsymbol{N}} \cdot \boldsymbol{\sigma}^{c}(\xi)+h \underline{\boldsymbol{b}}^{c}(\xi)=\underline{0} \rho_{\omega} \boldsymbol{\sigma}^{\omega}(\xi) \\
\sigma_{x y}^{s x}(\xi)=\sigma_{y y}^{s x}(\xi)=\sigma_{x x}^{s y}(\xi)=\sigma_{x y}^{s y}(\xi)=0
\end{array}\right.
$$

with the boundary conditions given at (4-11).

A closed-form solution exists for the localization problem (4-12). For concrete local stress field, it reads

$$
\left(\begin{array}{c}
\sigma_{x x}^{c}(\xi) \\
\sigma_{y y}^{c}(\xi) \\
\sigma_{x y}^{c}(\xi)
\end{array}\right)=\left(\begin{array}{c}
\frac{\cot \theta_{r}}{\rho_{c} h} N_{x y}-\frac{2 s_{r} \rho_{s x}}{\phi_{x} \rho_{c} \sin \theta_{r}} \int_{|\xi|}^{1} \tau_{x}(s) d s+g_{n}-\cot \theta_{r} g_{t} \\
\frac{\tan \theta_{r}}{\rho_{c} h} N_{x y}+\frac{2 s_{r} \rho_{s y}}{\phi_{y} \rho_{c} \cos \theta_{r}} \int_{|\xi|}^{1} \tau_{y}(s) d s+g_{n}+\tan \theta_{r} g_{t} \\
\frac{1}{\rho_{c} h} N_{x y}
\end{array}\right)
$$

while for steel local stress fields, one finds:

$$
\begin{aligned}
& \boldsymbol{\sigma}^{s x}(\xi)=\left[\frac{1}{\rho_{s x} h}\left(N_{x x}-\cot \theta_{r} N_{x y}\right)+\frac{2 s_{r}}{\phi_{x} \sin \theta_{r}} \int_{|\xi|}^{1} \tau_{x}(s) d s-\frac{\rho_{c}}{\rho_{s x}}\left(g_{n}-\cot \theta_{r} g_{t}\right)\right] \underline{e}_{x} \otimes \underline{e}_{x} \\
& \boldsymbol{\sigma}^{s y}(\xi)=\left[\frac{1}{\rho_{s y} h}\left(N_{y y}-\tan \theta_{r} N_{x y}\right)-\frac{2 s_{r}}{\phi_{y} \cos \theta_{r}} \int_{|\xi|}^{1} \tau_{y}(s) d s-\frac{\rho_{c}}{\rho_{s y}}\left(g_{n}+\tan \theta_{r} g_{t}\right)\right] \underline{e}_{y} \otimes \underline{e}_{y}
\end{aligned}
$$

\subsection{Local strain fields}

The concrete local strain field $\boldsymbol{\varepsilon}^{c}$ is simply obtained by applying the concrete isotropic elastic damage constitutive law defined in (3-1) to the local stress field (4-13): 


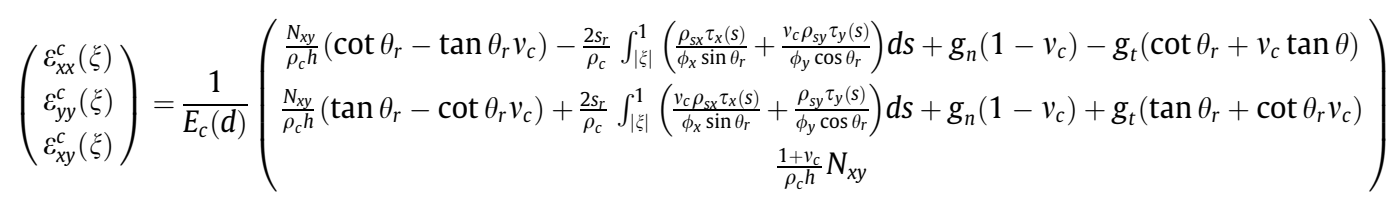

In a similar way, the strain fields of the steel bars $\varepsilon^{s x}$ and $\varepsilon^{s y}$ are obtained by applying the steel constitutive law (3-9) to the local stress fields (4-14), with the assumption (ensured by the threshold function defined in Section 5.3.4) that the steel behaves elastically between two successive cracks:

$$
\begin{aligned}
& \varepsilon_{x x}^{s x}(\xi)=\frac{1}{\rho_{s x} h E_{s}}\left(N_{x x}-\cot \theta_{r} N_{x y}\right)+\frac{2 s_{r}}{E_{s x} \phi_{x} \sin \theta_{r}} \int_{|\xi|}^{1} \tau_{x}(s) d s-\frac{\rho_{c}}{\rho_{s x} E_{s x}}\left(g_{n}-\cot \theta_{r} g_{t}\right) \\
& \varepsilon_{y y}^{s y}(\xi)=\frac{1}{\rho_{s y} h E_{s}}\left(N_{y y}-\tan \theta_{r} N_{x y}\right)-\frac{2 s_{r}}{E_{s y} \phi_{y} \cos \theta_{r}} \int_{|\xi|}^{1} \tau_{y}(s) d s-\frac{\rho_{c}}{\rho_{s y} E_{s y}}\left(g_{n}+\tan \theta_{r} g_{t}\right)
\end{aligned}
$$

\subsection{Compatibility of the average local strain fields}

The fourth step of the analytical multi-scale analysis presented in Section 2.1 consists in performing the averages of the local strain fields and to apply the following compatibility conditions:

(i) The generalized strain in the $\alpha$ direction is equal to the average local elastic strain of the $\alpha$ reinforcement steel bars $\varepsilon_{\alpha \alpha}^{s \alpha}$ between two consecutive cracks plus the $\alpha$ steel plastic strain $\varepsilon_{\alpha}^{p s}$ variable, localized at the crack crossing. Recall that no displacement discontinuity exists in the reinforcing steel bars and (2-2) is applied.

(ii) The generalized strain is equal to the sum of the concrete average strain and the crack displacement discontinuity equivalent strain, as expressed in (2-3).

These conditions can be written in the form of the following five scalar equations system:

$$
\left\{\begin{array}{l}
\epsilon_{x x}=\left\langle\varepsilon_{x x}^{s x}\right\rangle+\varepsilon_{x}^{p s} \\
\epsilon_{y y}=\left\langle\varepsilon_{y y}^{s y}\right\rangle+\varepsilon_{y}^{p s} \\
\boldsymbol{\epsilon}=\left\langle\boldsymbol{\varepsilon}^{c}\right\rangle+\boldsymbol{\varepsilon}^{r}
\end{array}\right.
$$

where $\boldsymbol{\varepsilon}^{r}$ is the crack displacement discontinuity equivalent strain field due to cracks corresponding to the second term on the right side of $(2-3)$ :

$$
\begin{aligned}
\boldsymbol{\varepsilon}^{r}\left(\underline{w}, \theta_{r}\right) & =\frac{1}{|\boldsymbol{\Omega}|} \int_{\Gamma_{r}} \underline{\llbracket} \rrbracket \otimes^{s} \underline{n} d S=\frac{1}{s_{r}}\left(\begin{array}{cc}
-w_{n} \sin \theta_{r}+w_{t} \cos \theta_{r} \\
w_{n} \cos \theta_{r}+w_{t} \sin \theta_{r}
\end{array}\right) \otimes^{s}\left(\begin{array}{c}
-\sin \theta_{r} \\
\cos \theta_{r}
\end{array}\right) \\
& =\frac{1}{2 s_{r}}\left(\begin{array}{cc}
2 w_{n} \sin ^{2} \theta_{r}-w_{t} \sin 2 \theta_{r} & -w_{n} \sin 2 \theta_{r}+w_{t} \cos 2 \theta_{r} \\
-w_{n} \sin 2 \theta_{r}+w_{t} \cos 2 \theta_{r} & 2 w_{n} \cos ^{2} \theta_{r}+w_{t} \sin 2 \theta_{r}
\end{array}\right)
\end{aligned}
$$

since the displacement discontinuity vector at cracks is defined as $\llbracket \underline{u} \rrbracket=\underline{w}=\left(w_{n}, w_{t}\right)$. The previous expression can be written as $\boldsymbol{\varepsilon}^{r}=\mathbb{M}^{\varepsilon w}\left(\theta_{r}\right) \cdot \underline{w}$, so that the third order tensor $\mathbb{M}^{\varepsilon w}\left(\theta_{r}\right)$ defines the geometrical relation between the crack displacement $\underline{w}$ and the crack displacement discontinuity equivalent strain $\boldsymbol{\varepsilon}^{r}$.

The average of the concrete strain field $\boldsymbol{\varepsilon}^{c}(4-15)$ is then calculated by means of (4-3):

$$
\left(\begin{array}{c}
\left\langle\varepsilon_{x x}^{c}\right\rangle \\
\left\langle\varepsilon_{y y}^{c}\right\rangle \\
\left\langle\varepsilon_{x y}^{c}\right\rangle
\end{array}\right)=\frac{1}{E_{c}(d)}\left(\begin{array}{c}
\frac{N_{x y}}{\rho_{c} h}\left(\cot \theta_{r}-v_{c} \tan \theta_{r}\right)-\frac{2 s_{r}}{\rho_{c}}\left(\frac{\rho_{s x} x_{x}^{o}}{\phi_{x} \sin \theta_{r}}+\frac{v_{c} \rho_{s y} \tau_{y}^{o}}{\phi_{y} \cos \theta_{r}}\right)+g_{n}\left(1-v_{c}\right)-g_{t}\left(\cot \theta_{r}+v_{c} \tan \theta\right) \\
\frac{N_{x y}}{\rho_{c} h}\left(\tan \theta_{r}-\cot \theta_{r} v_{c}\right)+\frac{2 s_{r}}{\rho_{c}}\left(\frac{v_{c} \rho_{s x} \tau_{x}^{0}}{\phi_{x} \sin \theta_{r}}+\frac{\rho_{s y} \tau_{y}^{o}}{\phi_{y} \cos \theta_{r}}\right)+g_{n}\left(1-v_{c}\right)+g_{t}\left(\tan \theta_{r}+\cot \theta_{r} v_{c}\right) \\
\frac{1+v_{c}}{\rho_{c} h} N_{x y}
\end{array}\right)
$$

where $\tau_{\alpha}^{o}$ is the average tension stiffening effect:

$$
\tau_{\alpha}^{o}=\left\langle\int_{|\xi|}^{1} \tau_{\alpha}(z) d z\right\rangle=\frac{1}{2} \int_{-1}^{1} \int_{|\xi|}^{1} \tau_{\alpha}(z) d z d \xi
$$

As shown in (A-5) of Appendix A, the average tension stiffening bond stress $\tau_{x}^{0}$ and $\tau_{y}^{o}$ is proportional to the $x$ and $y$ steelconcrete slips $v_{x}$ and $v_{y}$. Following the generalization of (3-8), this proportionality is modified in order to take into account the inelastic slip variables $v_{x}^{p}$ and $v_{y}^{p}$ :

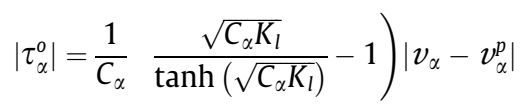


The coefficient $C_{\alpha}$ is given by:

$$
C_{\alpha}=s_{r, \alpha}^{2} \frac{\left(1+\frac{\rho_{s x} E_{s}}{\rho_{c} E_{c}}\right)}{\phi_{\alpha} E_{s}}
$$

where $s_{r, x}=s_{r} /\left|\sin \theta_{r}\right|$ and $s_{r, y}=s_{r} / \cos \theta_{r}$ are the crack spacings seen by the $x$ and $y$ steel reinforcement bars respectively, see Fig. 4-1(a).

Taking into account the definition (4-20) and Fig. 4-1(b), in tension (positive values of the difference $v_{\alpha}-v_{\alpha}^{p}$ ) $\tau_{x}^{o}$ has an opposite sign to $\theta_{r}$ while $\tau_{y}^{o}$ is always positive. Therefore, the global bond-stiffness tensor postulated in (3-7) is defined as:

$$
\left.\boldsymbol{K}^{\tau}=-\operatorname{sign}\left(\theta_{r}\right) \frac{1}{C_{x}} \frac{\sqrt{C_{x} K_{l}}}{\tanh \left(\sqrt{\left.C_{x} K_{l}\right)}\right.}-1\right) \underline{e}_{x} \otimes \underline{e}_{x}+\frac{1}{C_{y}}\left(\frac{\sqrt{C_{y} K_{l}}}{\tanh \left(\sqrt{C_{y} K_{l}}\right)}-1\right) \underline{e}_{y} \otimes \underline{e}_{y}
$$

In a similar manner to the average concrete strain fields, the average strain fields of the steel bars in $x$ and $y$ direction are obtained by applying (4-3) to the local strain fields (4-16):

$$
\begin{aligned}
& \left\langle\varepsilon_{x x}^{s x}\right\rangle=\frac{1}{\rho_{s x} h E_{s}}\left(N_{x x}-\cot \theta_{r} N_{x y}\right)+\frac{2 s_{r} \tau_{x}^{o}}{E_{s} \phi_{x} \sin \theta_{r}}-\frac{\rho_{c}}{\rho_{s x} E_{s}}\left(g_{n}-\cot \theta_{r} g_{t}\right) \\
& \left\langle\varepsilon_{y y}^{s y}\right\rangle=\frac{1}{\rho_{s y} h E_{s}}\left(N_{y y}-N_{x y} \tan \theta_{r}\right)-\frac{2 s_{r} \tau_{y}^{o}}{E_{s} \phi_{y} \cos \theta_{r}}-\frac{\rho_{c}}{\rho_{s y} E_{s}}\left(g_{n}+\tan \theta_{r} g_{t}\right)
\end{aligned}
$$

\subsection{Results of the analytical multi-scale analysis}

The solution of the five equations system (4-17), using definitions (4-19) and (4-24), defines the stress resultant $\boldsymbol{N}$ and the two components of the concrete stress at cracks $g$ as a function of the generalized strain $\epsilon$ and the internal variables describing the retained physical phenomena.

\subsubsection{Stress resultant $\mathbf{N}$}

The stress resultant $\boldsymbol{N}$ can be expressed as the sum of the average steel and concrete stresses, since $\underline{\varepsilon}^{p s}=\left(\varepsilon_{x}^{p s}, \varepsilon_{y}^{p s}\right)$ and $\varepsilon^{r}$ act as the average « inelastic » strain variables for each of the components respectively:

$$
\boldsymbol{N}=\rho_{s x} h E_{s}\left(\epsilon_{x x}-\varepsilon_{x}^{p s}\right) \underline{e}_{x} \otimes \underline{e}_{x}+\rho_{s y} h E_{s}\left(\epsilon_{y y}-\varepsilon_{y}^{p s}\right) \underline{e}_{y} \otimes \underline{e}_{y}+\rho_{c} h \mathbb{C}_{c}(d):\left(\boldsymbol{\epsilon}-\boldsymbol{\varepsilon}^{r}\right)
$$

The constitutive law (4-25) can be explicitly expressed as a function of the variables $\epsilon, \underline{w}, \underline{\varepsilon}^{p s}$ and $d$ :

$$
\boldsymbol{N}=\mathbb{A}(d): \boldsymbol{\epsilon}-\mathbb{B}\left(d, \theta_{r}\right) \cdot \underline{w}-\mathbb{C} \cdot \underline{\varepsilon}^{p s}
$$

The fourth order positive symmetric tensor $\mathbb{A}(d)$ and the third order tensors $\mathbb{B}(d)$ and $\mathbb{C}$ read:

$$
\begin{aligned}
& A(d)=\rho_{c} h \mathbb{C}_{c}(d)+\sum_{\alpha} \rho_{s \alpha} h E_{s} \underline{e}_{\alpha} \otimes \underline{\boldsymbol{e}}_{\alpha} \otimes \underline{\boldsymbol{e}}_{\alpha} \otimes \underline{\boldsymbol{e}}_{\alpha} \\
& B\left(d, \theta_{r}\right)=\rho_{c} h \mathbb{C}_{c}(d): \mathbb{M} \mathbb{M}^{\varepsilon w}\left(\theta_{r}\right) \\
& C=\sum_{\alpha} \rho_{s \alpha} h E_{s} \underline{e}_{\alpha} \otimes \underline{e}_{\alpha} \otimes \underline{e}_{\alpha}
\end{aligned}
$$

\subsubsection{Concrete stress at cracks $g$ and average tension stiffening stress in concrete $\boldsymbol{\sigma}^{\tau}$}

The solution of (4-17) for the concrete stress at cracks $\underline{g}$ that ensures the displacements compatibility is:

$$
\underline{g}=\left[\mathbb{C}_{c}(d):\left(\boldsymbol{\epsilon}-\boldsymbol{\varepsilon}^{r}\right)-\boldsymbol{\sigma}^{\tau}\right]_{n n, t n}
$$

which is the $n n$ and $t n$ components of the difference of the average concrete stress and the average tension stiffening stress in concrete $\boldsymbol{\sigma}^{\tau}$ due to the bond stress:

$$
\begin{aligned}
\sigma_{x x}^{\tau} & =-\frac{2 s_{r} \rho_{s x}}{\rho_{c} \phi_{x} \sin \theta_{r}} \tau_{x}^{o}=-\frac{2 s_{r} \rho_{s x}}{\rho_{c} \phi_{x} \sin \theta_{r}} K_{x x}^{\tau}\left(v_{x}-v_{x}^{p}\right)=\frac{2 s_{r, x} \rho_{s x}}{\rho_{c} \phi_{x}}\left|K_{x x}^{\tau}\right|\left(v_{x}-v_{x}^{p}\right) \\
\sigma_{y y}^{\tau} & =\frac{2 s_{r} \rho_{s y}}{\rho_{c} \phi_{y} \cos \theta_{r}} \tau_{y}^{o}=\frac{2 s_{r} \rho_{s y}}{\rho_{c} \phi_{y} \cos \theta_{r}} K_{y y}^{\tau}\left(v_{y}-v_{y}^{p}\right)=\frac{2 s_{r, y} \rho_{s y}}{\rho_{c} \phi_{y}}\left|K_{y y}^{\tau}\right|\left(v_{y}-v_{y}^{p}\right) \\
\sigma_{x y}^{\tau} & =0
\end{aligned}
$$




\section{Thermodynamic formulation of the global model for RC panels}

The Helmholtz free energy surface density is derived from the results of the multi-scale analysis of the previous section. This function is adopted to define the thermodynamic forces associated with the internal variables $\underline{w}, \underline{v}^{p}, \underline{e}^{p s}$ and $d$ and the observable variable $\epsilon$ through the state equations. Then, the chosen threshold functions describing the internal state variable evolution are presented.

\subsection{Helmholtz free energy surface density}

The Helmholtz free energy surface density of the model is defined as the sum of two different Helmholtz free energy sources, associated to two different types of stress in the RVE: the steel and concrete average stresses and the tension stiffening stress in concrete (see the three springs of the rheological model of Fig. 5-1). The free energy associated to the average stresses is obtained by integration of the constitutive law (4-25) with respect to the generalized strain $\epsilon$ :

$$
\psi_{1}^{0}=\frac{1}{2} \rho_{s x} h E_{s}\left(\epsilon_{x x}-\varepsilon_{x}^{p s}\right)^{2}+\frac{1}{2} \rho_{s y} h E_{s}\left(\epsilon_{y y}-\varepsilon_{y}^{p s}\right)^{2}+\frac{1}{2} \rho_{c} h\left(\boldsymbol{\epsilon}-\boldsymbol{\varepsilon}^{r}\right): \mathbb{C}_{c}(\boldsymbol{d}):\left(\boldsymbol{\epsilon}-\boldsymbol{\varepsilon}^{r}\right)
$$

The free energy associated to the tension stiffening stress in concrete is obtained by integrating the stress (4-29) with respect to $\underline{v}=\boldsymbol{M}^{\nu w}\left(\theta_{r}\right) \cdot \underline{w}$ and averaging the $x$ term over $s_{r, x} /\left(\rho_{c} h\right)$ and the $y$ term over $s_{r, y} /\left(\rho_{c} h\right)$ :

$$
\psi_{2}^{o}=\frac{1}{2}\left(\boldsymbol{M}^{v w}\left(\theta_{r}\right) \cdot \underline{w}-\underline{v}^{p}\right): \boldsymbol{K}^{s}\left(d, \theta_{r}\right):\left(\boldsymbol{M}^{v w}\left(\theta_{r}\right) \cdot \underline{w}-\underline{v}^{p}\right)
$$

with:

$$
K_{x x}^{s}=\frac{2 s_{r, x} \rho_{s x}}{\rho_{c} \phi_{x}}\left|K_{x x}^{\tau}\right| \frac{\rho_{c} h}{s_{r, x}}=\frac{2 \rho_{s x} h}{\phi_{x}}\left|K_{x x}^{\tau}\right| \quad K_{y y}^{s}=\frac{2 s_{r, y} \rho_{s y}}{\rho_{c} \phi_{y}}\left|K_{y y}^{\tau}\right| \frac{\rho_{c} h}{s_{r, y}}=\frac{2 \rho_{s y} h}{\phi_{y}}\left|K_{y y}^{\tau}\right|
$$

The total Helmholtz free energy surface density $\psi^{0}$ is the sum of the previous two sources of energy $\psi^{0}=\psi_{1}^{0}+\psi_{2}^{0}$ and is explicitly expressed as a function of the state variables $\epsilon, \underline{w}, \underline{v}^{p}, \underline{\varepsilon}^{p s}, d$ and the parameter $\theta_{r}$ :

$$
\begin{aligned}
\psi^{o}\left(\boldsymbol{\epsilon}, \underline{w}, \underline{v}^{p}, \underline{\varepsilon}^{p s}, d ; \theta_{r}\right)= & \frac{1}{2} \boldsymbol{\epsilon}: \mathbb{A}(d): \boldsymbol{\epsilon}-\boldsymbol{\epsilon}: \mathbb{B}\left(d, \theta_{r}\right) \cdot \underline{w}-\boldsymbol{\epsilon}: \mathbb{C} \cdot \underline{\varepsilon}^{p s}+\frac{1}{2} \underline{w} \cdot \boldsymbol{D}\left(d, \theta_{r}\right) \cdot \underline{w} \\
& +\frac{1}{2} \underline{v}^{p} \cdot \boldsymbol{E}\left(d, \theta_{r}\right) \cdot \underline{v}^{p}+\frac{1}{2} \underline{\varepsilon}^{p s} \cdot \boldsymbol{F} \cdot \underline{\varepsilon}^{p s}-\underline{w} \cdot \boldsymbol{G}\left(d, \theta_{r}\right) \cdot \underline{v}^{p}
\end{aligned}
$$

where $\boldsymbol{D}\left(d, \theta_{r}\right), \boldsymbol{E}(d), \boldsymbol{F}$ and $\boldsymbol{G}\left(d, \theta_{r}\right)$ are the following second order tensors:

$$
\begin{aligned}
& \boldsymbol{D}\left(d, \theta_{r}\right)=\rho_{c} h^{t} \mathbb{M}^{\varepsilon w}\left(\theta_{r}\right): \mathbb{C}_{c}(d): \mathbb{M}^{\varepsilon w}\left(\theta_{r}\right)+{ }^{t} \boldsymbol{M}^{v w}\left(\theta_{r}\right) \cdot \boldsymbol{K}^{s}\left(d, \theta_{r}\right) \cdot \boldsymbol{M}^{v w}\left(\theta_{r}\right) \\
& \boldsymbol{E}(d)=\boldsymbol{K}^{s}\left(d, \theta_{r}\right) \\
& \boldsymbol{F}=\sum_{\alpha} \rho_{s \alpha} h E_{s} \underline{e}_{\alpha} \otimes \underline{e}_{\alpha} \\
& \boldsymbol{G}\left(d, \theta_{r}\right)={ }^{t} \boldsymbol{M}^{v w}\left(\theta_{r}\right) \cdot \boldsymbol{K}^{s}\left(d, \theta_{r}\right)
\end{aligned}
$$

and the generic component $i j k$ of the transposed tensor ${ }^{t} \mathbb{M}^{\varepsilon w}$ reads ${ }^{t} \mathbb{M}_{i j k}^{\varepsilon w}:=\mathbb{M}_{k i j}^{E w}$.

The parametric dependence on $\theta_{r}$ is not explicitly indicated hereinafter in order to simplify the notation.

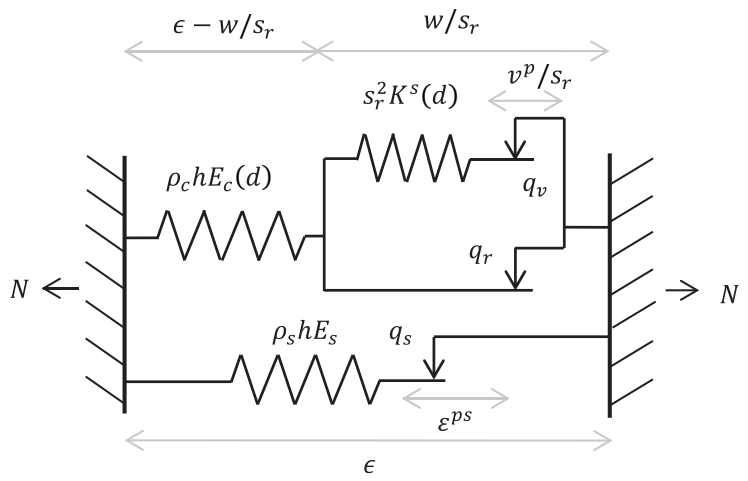

Fig. 5-1. One-dimensional rheological scheme of the proposed global constitutive model. 


\subsection{State equations}

By definition, the state equations (1-1) are obtained by derivation of the Helmholtz free energy surface density (5-4) with respect to the state variables. In particular, the constitutive law (4-26) is retrieved by derivation of $\psi^{0}$ with respect to the generalized strain tensor $\epsilon$ :

$$
\boldsymbol{N}=\frac{\partial \psi^{0}}{\partial \boldsymbol{\epsilon}}=\mathbb{A}(d): \boldsymbol{\epsilon}-\mathbb{B}(d) \cdot \underline{w}-\mathbb{C} \cdot \underline{\varepsilon}^{p s}
$$

The (dissipative) energy release rate is calculated as the derivative of $\psi^{\circ}$ with respect to the damage variable $d$ :

$$
-Y=\frac{\partial \psi^{0}}{\partial d}=\frac{1}{2} \boldsymbol{\epsilon}: \mathbb{A}^{\prime}(d): \boldsymbol{\epsilon}-\boldsymbol{\epsilon}: \mathbb{B}^{\prime}(d) \cdot \underline{w}+\frac{1}{2} \underline{w} \cdot \boldsymbol{D}^{\prime}(d) \cdot \underline{w}+\frac{1}{2} \underline{v}^{p} \cdot \boldsymbol{E}^{\prime}(d) \cdot \underline{v^{p}}-\underline{w} \cdot \boldsymbol{G}^{\prime}(d) \cdot \underline{v}^{p}
$$

where $\mathbb{A}^{\prime}(d)$ stands for the derivative of $\mathbb{A}(d)$ with respect to $d$ (and equivalently for the other tensors).

Analogously, the thermodynamic force associated with $\underline{w}$, which is hereinafter called macro concrete stress at cracks, is defined as:

$$
-\underline{q_{r}}=\frac{\partial \psi^{0}}{\partial \underline{w}}=-\boldsymbol{\epsilon}: \mathbb{B}(d)+\boldsymbol{D}(d) \cdot \underline{w}-\boldsymbol{G}(d) \cdot \underline{v}^{p}
$$

The thermodynamic force associated with $\underline{v}^{p}$, named macro bond-slip stress, is calculated as:

$$
-\underline{q}_{v}=\frac{\partial \psi^{o}}{\partial \underline{v}^{p}}=\boldsymbol{E}(d) \cdot \underline{v}^{p}-\underline{w} \cdot \boldsymbol{G}(d)
$$

Finally, the thermodynamic force associated with $\underline{\varepsilon}^{p s}$, which is hereinafter called macro steel stress, reads:

$$
-\underline{q}_{s}=\frac{\partial \psi^{0}}{\partial \underline{\varepsilon}^{p s}}=-\boldsymbol{\epsilon}: \mathbb{C}+\boldsymbol{F} \cdot \underline{\varepsilon}^{p s}
$$

In the one-dimensional case, the developed constitutive model can be represented by the rheological scheme of Fig. 5-1, where it can be seen that the stress resultant $\boldsymbol{N}$ is the sum of steel and concrete contributions by means of their average stress.

\subsection{Threshold surfaces and flow rules}

The evolution of the four internal variables of the model $\left(d, \underline{w}, \underline{v}^{p}, \underline{\varepsilon}^{p s}\right)$ is defined by means of the threshold functions $f_{i}$ $(i=d, g n, g t, v x, v y, s x, s y)$ and the associated plastic multipliers $\dot{\lambda}_{i}$, by means of the normality rule (1-3), following the principles of GSM theory.

\subsubsection{Concrete damage}

Concrete damage is controlled by the limitation of the energy release rate in the concrete section $\rho_{c} h$ by means of a constant threshold $k_{0}$ :

$$
f_{d}(Y)=Y-\rho_{c} h k_{0} \leqslant 0
$$

The flow of the damage variable is given by the normality rule:

$$
\dot{d}=\dot{\lambda}_{d} \frac{\partial f_{d}}{\partial Y}=\dot{\lambda}_{d}
$$

where $f_{d}$ and $\dot{\lambda}_{d}$ satisfy the Kuhn-Tucker conditions (1-4).

\subsubsection{Concrete cracking}

As demonstrated in Appendix C, the concrete stress at cracks (4-28) and the macro concrete stress at cracks (5-8) can be directly related by:

$$
\underline{g}=\left(g_{n}, g_{t}\right)=\underline{q_{r}} \frac{s_{r}}{\rho_{c} h}
$$

This result is of great importance since it allows defining the threshold surfaces for the macro concrete stress at cracks $\underline{q}_{r}$ and the flow of its associated internal variable crack displacement $\underline{w}$ using well known physical mechanisms concerning concrete stress at cracks $\underline{g}$.

In the normal direction, the bridging stress phenomenon is retained to describe the evolution of the normal crack displacement $w_{n}$ as a function of the normal stress $g_{n}$ (or thermodynamic force $q_{r, n}$ ). In the tangential direction, aggregate interlock (see [31]) governs the flow of tangential crack displacement $w_{t}$ as a function of the concrete tangential stress at cracks $g_{t}$ 
(or thermodynamic force $q_{r, t}$ ). After crack onset, which is defined by the Rankine criterion (3-3), both phenomena are activated and evolve following the threshold functions defined in this section.

It is noted that other phenomena which affect concrete stress at cracks, like dilatancy stress or dowel effect, are neglected in the model as a first approximation since it is considered that their influence on results is of second order when compared to bridging stress and aggregate interlock. In particular, the choice of neglecting the dowel effect is in accordance with the steel reinforcement bars constitutive equation (3-9), which assumes that they only carry stress along their axis and do not offer any shear nor bending resistance.

5.3.2.1. Normal crack opening: bridging stress. The definition of the bridging stress law, or the relationship between the normal stress $g_{n}$ and the normal crack displacement $w_{n}$, is based on the following principles, which are also graphically shown in Fig. 5-2:

- The envelope (blue) curve $G_{n}\left(w_{n}\right)$ is related to an energy dissipation equal to the fracture energy:

$$
\int_{0}^{+\infty} G_{n}\left(w_{n}\right) d w_{n}=G_{f}
$$

- The envelope curve is equal to $f_{c t}$ before concrete cracking $G_{n}(0)=f_{c t}$, in order to be in accordance with the Rankine criterion (3-3).

- Unloading (red curve) is done elastically (constant crack opening) until compressive stresses are reached. Under compressive stress, crack recloses with an unloading slope equal to $\alpha_{u} E_{c}$ until a crack width reclosing value equal to $\alpha_{r} w_{n}^{\max }$, where $\alpha_{u}$ accounts for the crack unload slope ratio, $w_{n}^{\max }$ is the historical maximum crack opening, $\alpha_{r}$ is the crack reclosing ratio.

- Reloading (green curve) is done elastically (constant crack opening) until tensile stresses are reached; at this moment, crack reopens and the envelope curve is attained at the point corresponding to the unloading onset.

Following the previous principles, the threshold function for the thermodynamic force $q_{r, n}$ is defined by the previously defined bridging stress law using the transformation (5-13):

$$
f_{g n}\left(q_{r, n} ; \underline{w} ; w_{n}^{\max }\right)=\max _{i=1,2}\left(f_{g n i}\left(q_{r, n} ; w_{n} ; w_{n}^{\max }\right)\right) \leqslant 0
$$

with:

$$
\begin{aligned}
& f_{g n 1}\left(q_{r, n} ; w_{n} ; w_{n}^{\max }\right)=q_{r, n}-\frac{\rho_{c} h}{s_{r}} G_{n}\left(w_{n}^{\max }\right) \frac{w_{n}}{w_{n}^{\max }} \\
& f_{\text {gn2 }}\left(q_{r, n} ; w_{n} ; w_{n}^{\max }\right)=\left(-q_{r, n}+\frac{\rho_{c} h}{s_{r}} \alpha_{u} E_{c} \frac{w_{n}^{\max }-w_{n}}{s_{r}}\right) \mathcal{H}\left(w_{n}-\alpha_{r} w_{n}^{\max }\right)
\end{aligned}
$$

where $\mathcal{H}$ stands for the Heaviside function, and $G_{n}\left(w_{n}\right)$ is the nonnegative bridging stress envelope curve depending on the crack opening of a plain concrete tensile test, which is retained as linear:

$$
G_{n}\left(w_{n}\right)= \begin{cases}f_{c t}-\frac{f_{c t}^{2}}{2 G_{f}} w_{n} & 0 \leqslant w_{n} \leqslant \frac{2 G_{f}}{f_{c t}} \\ 0 & \frac{2 G_{f}}{f_{c t}} \leqslant w_{n}\end{cases}
$$

which respects the condition corresponding to the concrete fracture energy $G_{f}(5-14)$.

(a)

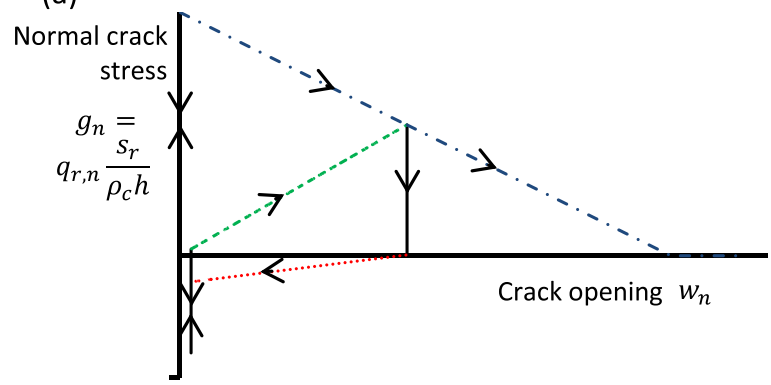

(b)

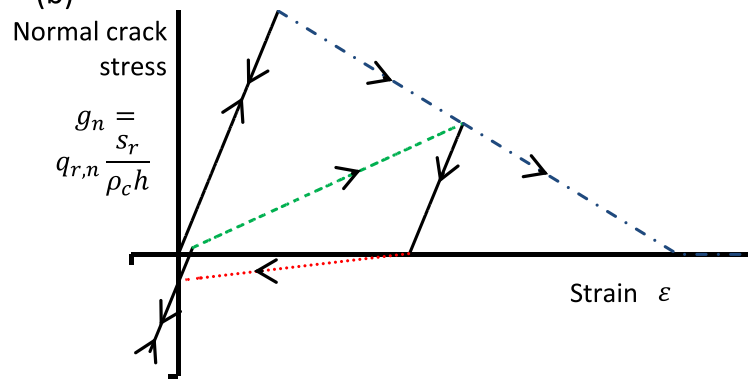

Fig. 5-2. Normal-to-crack concrete stress at cracks $g_{n}$ as a function of (a) crack opening $w_{n}$ and (b) average strain in the concrete member; continous line $=$ elastic phase, dotted-dashed line $=f_{g_{n 1}}$ with $w_{n}=w_{n}^{\max }$, dashed line $=f_{g_{n 1}}$ with $w_{n}<w_{n}^{\max }$, dotted line $=f_{g_{n 2}}$. 
The flow of the crack opening $\dot{w}_{n}$ is given by the normality rule:

$$
\dot{w}_{n}=\dot{\lambda}_{g n} \frac{\partial f_{g n}}{\partial q_{r, n}}= \begin{cases}\dot{\lambda}_{g n} & \text { if } f_{g n}=f_{g n 1} \\ -\dot{\lambda}_{g n} & \text { if } f_{g n}=f_{g n 2}\end{cases}
$$

where $f_{g n}$ and $\dot{\lambda}_{g n}$ satisfy the Kuhn-Tucker conditions (1-4).

5.3.2.2. Tangential crack displacement: aggregate interlock. The evolution of the tangential crack displacement $w_{t}$ as a function of the tangential concrete stress $g_{t}$ at cracks is governed by the retained aggregate interlock law of Fig. 5-3(b), which is symmetric in both senses of loading and has an initial stress threshold $T_{o}$ for the tangential crack displacement onset. These choices aim at reproducing some of the observed phenomena on the experimental curve of Fig. 5-3(a):

- The loading stress - tangential displacement curve is roughly linear for common tangential displacement values in RC structures $w_{t} \leqslant 0.5 \mathrm{~mm}$.

- The experimental unloading is characterized by a relatively small variation of the tangential displacement $w_{t}$ until 0 tangential stress is reached.

- The cyclic response is symmetric in both tangential loading directions.

It is noted that, in a first approximation, no coupling of the retained aggregate interlock law with the normal concrete stress at cracks $g_{n}$ has been considered, in order to minimize coupling between the different nonlinear phenomena and so to improve the robustness of the numerical integration of the model, which has to deal with the evolution of many internal variables at the same time. However, this coupling is partly taken into account by an adapted choice of $T_{o}$ and $T_{1}$ parameters, see Appendix D.

Using the transformation (5-13), the threshold surface of Fig. 5-3(b) for the thermodynamic force $q_{r, t}$ as a function of the tangential crack displacement $w_{t}$ is defined as:

$$
f_{g t}\left(q_{r, t} ; w_{t}\right)=\max _{i=1,2}\left(f_{g t i}\left(q_{r, t} ; w_{t}\right)\right) \leqslant 0
$$

with:

$$
\begin{aligned}
& f_{g t 1}\left(q_{r, t} ; w_{t}\right)=\left(q_{r, t}-\frac{\rho_{c} h}{s_{r}}\left(T_{o}+T_{1} w_{t}\right)\right) \mathcal{H}\left(q_{r, t} w_{t}\right) \\
& f_{\text {gt } 2}\left(q_{r, t} ; w_{t}\right)=-q_{r, t} \operatorname{sign}\left(w_{t}\right)
\end{aligned}
$$

The flow of the crack tangential displacement $w_{t}$ is then:

$$
\dot{w}_{t}=\dot{\lambda}_{g t} \frac{\partial f_{g t}}{\partial q_{r, t}}= \begin{cases}\dot{\lambda}_{g t} & \text { if } f_{g t}=f_{g_{t 1}} \\ -\dot{\lambda}_{g t} & \text { if } f_{g t}=f_{g_{t 2}}\end{cases}
$$

where $f_{g t}$ and $\dot{\lambda}_{\text {gt }}$ satisfy the Kuhn-Tucker conditions (1-4).

\subsubsection{Steel-concrete bond-slip}

Steel-concrete bond slip is defined by its main effect at the global scale, the well-known tension stiffening phenomenon. Two main principles are used for the definition of the threshold function associated to $\underline{q}_{v}$ :

(a)

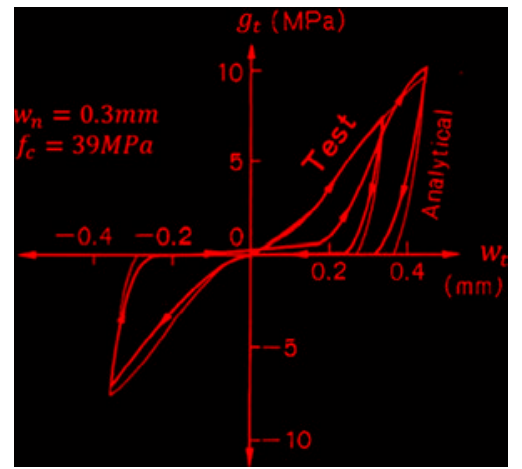

(b) Tangential crack stress

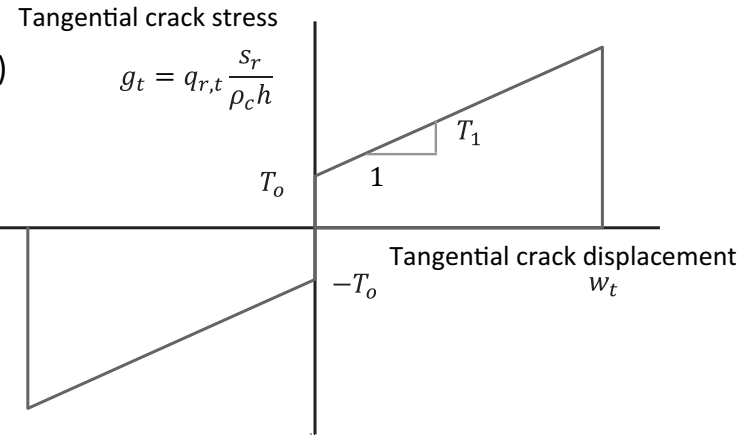

Fig. 5-3. (a) Experimental and analytical results of Contact Density Model [19] and (b) retained simplified law for the description of aggregate interlock. 
- A stress in concrete equal to $k_{\mathrm{t}} f_{c t}$ is supposed to be created when the tension stiffening is fully mobilized in tension loadings, in order to reproduce the MC10 tension stiffening effect considered for the crack opening calculation.

- Tension stiffening effect has the same absolute value and an opposite sign when unloading, in order to reproduce the negative tension stiffening identified e.g. by Zanuy et al. [32].

According these two considerations, for the both directions of reinforcement $\alpha$, the absolute value of the average tension stiffening stress in concrete is limited to $k_{t} f_{c t}$ :

$$
\left|\sigma_{\alpha \alpha}^{\tau}\right| \leqslant k_{t} f_{c t}
$$

The tension stiffening stress can be expressed as a function of the macro bond-slip stress, by considering the definition of $\sigma_{\alpha \alpha}^{\tau}$ given by (4-29):

$$
\sigma_{\alpha \alpha}^{\tau}=\frac{2 s_{r, \alpha} \rho_{s \alpha}}{\rho_{c} \phi_{\alpha}}\left|K_{\alpha \alpha}^{\tau}\right|\left(v_{\alpha}-v_{\alpha}^{p}\right)=\frac{s_{r, \alpha}}{\rho_{c} h}\left|K_{\alpha \alpha}^{s}\right|\left(v_{\alpha}-v_{\alpha}^{p}\right)=\frac{s_{r, \alpha}}{\rho_{c} h} q_{v, \alpha}
$$

Therefore, the threshold can be defined from (5-22) and (5-23):

$$
f_{v \alpha}\left(q_{v, \alpha}\right)=\left|q_{v, \alpha}\right|-\frac{\rho_{c} h}{s_{r, \alpha}} k_{t} f_{c t} \leqslant 0
$$

The evolution of the inelastic steel-concrete slip internal variable is defined by the normality rule:

$$
\dot{\nu}_{\alpha}^{p}=\dot{\lambda}_{v \alpha} \frac{\partial f_{v \alpha}}{\partial q_{v, \alpha}}=\dot{\lambda}_{v \alpha} \operatorname{sign}\left(q_{v, \alpha}\right)
$$

where $f_{v \alpha}$ and $\dot{\lambda}_{v \alpha}$ satisfy the Kuhn-Tucker conditions (1-4).

\subsubsection{Yielding of steel reinforcement bars at crack crossings}

Expressions (4-14) show that, when the RVE is submitted to tension (related to positive values of $v_{x}$ and $v_{y}$ ), the maximum stress in steel reinforcement bars is attained for $\xi= \pm 1$ (at cracks location) both for $x$ and $y$ bars, since $\tau_{x}^{o}(s) \sin \theta_{r} \leqslant 0$ and $\tau_{y}^{o}(s) \cos \theta_{r} \geqslant 0$ from (4-23) and (3-8). As the retained model for bond stresses takes into account the negative tension stiffening (inversion of the sign of the bond stresses $\tau_{\alpha}$ ), the same reasoning can be made for the minimum steel stress in compression. The steel stresses at cracks are calculated from (4-14) as:

$$
\begin{aligned}
& \sigma^{s x}( \pm 1)=\left(\sigma_{x x}^{s x}-\frac{2 s_{r}}{\phi_{x} \sin \theta_{r}} \tau_{x}^{o}\right) \underline{e}_{x} \otimes \underline{e}_{x} \\
& \left.\sigma^{s y}( \pm 1)=\sigma_{y y}^{s y}+\frac{2 s_{r}}{\phi_{y} \cos \theta_{r}} \tau_{y}^{o}\right) \underline{e}_{y} \otimes \underline{e}_{y}
\end{aligned}
$$

The previous equations can also be expressed as a function of the thermodynamic force $\underline{q}_{s}$ and the internal variables $\underline{w}$ and $\underline{v}^{p}$ :

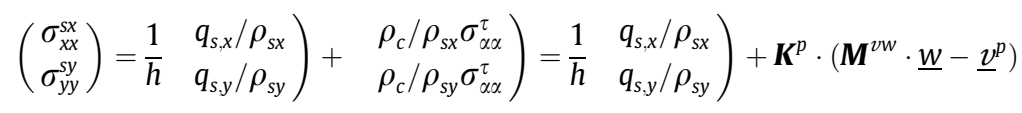

where $\boldsymbol{K}^{p}$ is the second order diagonal tensor defined by:

$$
\boldsymbol{K}^{p}=\sum_{\alpha} \frac{s_{r, \alpha}}{\rho_{s \alpha} h} K_{\alpha \alpha}^{s} \underline{e}_{\alpha} \otimes \underline{\boldsymbol{e}}_{\alpha}=\sum_{\alpha} \frac{2 s_{r, \alpha}}{\phi_{\alpha}}\left|K_{x x}^{\tau}\right| \underline{e}_{\alpha} \otimes \underline{\boldsymbol{e}}_{\alpha}
$$

Since the multi-scale procedure of Section 4 is performed under the assumption of linear elastic behavior of the steel reinforcement bars in the RVE, it is necessary to limit the steel yielding to the boundaries of the RVE, at the crack crossing, see Section 3.4. This is ensured by imposing a constant yield limit equal to $f_{s y}$ :

$$
f_{s \alpha}\left(q_{s, \alpha}, \underline{w}_{1}, \underline{w}_{2}, \underline{v}^{p}\right)=\left|q_{s, \alpha}+s_{r, \alpha}\left(\boldsymbol{K}^{s} \cdot\left(\boldsymbol{M}^{\nu w} \cdot \underline{w}-\underline{v}^{p}\right)\right) \cdot \underline{e}_{\alpha}\right|-\rho_{s \alpha} h f_{s y} \leqslant 0
$$

The evolution of the internal variables of steel plastic strain is defined by the normality rule:

$$
\dot{\varepsilon}_{\alpha}^{p s}=\dot{\lambda}_{s \alpha} \frac{\partial f_{s \alpha}}{\partial q_{s, \alpha}}=\dot{\lambda}_{s \alpha} \operatorname{sign}\left(q_{s, \alpha}+s_{r, \alpha}\left(\boldsymbol{K}^{s} \cdot\left(\boldsymbol{M}^{v w} \cdot \underline{w}-\underline{v}^{p}\right)\right) \cdot \underline{e}_{\alpha}\right)
$$

with $f_{s \alpha}$ and $\dot{\lambda}_{s \alpha}$ satisfying the Kuhn-Tucker conditions (1-4).

\subsubsection{Thermodynamic admissibility}

With the previous definitions of the thermodynamic forces, the power dissipation surface density $\dot{D}$ given by (1-2) reads: 


$$
\dot{D}=Y \dot{d}+\underline{q}_{r} \cdot \underline{\dot{w}}+\underline{q}_{v} \cdot \underline{\dot{\nu}}^{p}+\underline{q}_{s} \cdot \underline{\dot{\varepsilon}}^{p s}
$$

which has to be proven to be nonnegative for any load history. It has been chosen to prove the sufficient (but not necessary) condition of having non-negativity of all of the terms of the previous equation.

The first term of (5-31) is the dissipation rate associated with the damage evolution:

$$
Y \dot{d}=Y \dot{\lambda}_{d} \geqslant 0
$$

since $Y \geqslant 0$.

The second term of the power dissipation surface density is the power dissipated by crack evolution:

$$
\underline{q_{r}} \cdot \underline{\dot{w}}=q_{r, n} \dot{w}_{n}+q_{r, t} \dot{w}_{t}=q_{r, n} \dot{\lambda}_{g n} \operatorname{sign}\left(q_{r, n}\right)+q_{r, t} \dot{\lambda}_{g t} \operatorname{sign}\left(q_{r, t}\right)=\dot{\lambda}_{g n}\left|q_{r, n}\right|+\dot{\lambda}_{g t}\left|q_{r, t}\right| \geqslant 0
$$

The third term of $\dot{D}$ is the power dissipated by inelastic slip between concrete and steel bars:

$$
\underline{q}_{v} \cdot \underline{\dot{v}}^{p}=\sum_{\alpha} q_{v, \alpha} \dot{\lambda}_{v \alpha} \operatorname{sign}\left(q_{v, \alpha}\right)=\sum_{\alpha} \dot{\lambda}_{v \alpha}\left|q_{v, \alpha}\right| \geqslant 0
$$

Finally, the fourth term of (5-31) provides the power dissipated by yielding of steel reinforcing bars at crack crossings:

$$
\underline{q}_{s} \cdot \underline{\dot{\varepsilon}}^{p s}=\sum_{\alpha} q_{s, \alpha} \dot{\lambda}_{s \alpha} \operatorname{sign}\left(q_{s, \alpha}+s_{r, \alpha}\left(\boldsymbol{K}^{s} \cdot\left(\boldsymbol{M}^{v w} \cdot \underline{w}-\underline{v}^{p}\right)\right) \cdot \underline{e}_{\alpha}\right) \geqslant 0
$$

This condition is fulfilled if both terms of the summation in (5-35) are nonnegative. This is for all possible loading histories provided that (see demonstration in Appendix B)

$$
k_{t} \leqslant \frac{\rho_{s \alpha}}{\rho_{c}} \frac{f_{s y}}{f_{c t}} \quad \alpha=x, y
$$

Finally, combining (5-32)-(5-35) it can be concluded that the developed model is thermodynamically admissible since the total power dissipation surface density is nonnegative:

$$
\dot{D}=Y \dot{d}+\underline{q}_{r} \cdot \underline{\dot{w}}+\underline{q}_{v} \cdot \underline{\dot{\nu}}^{p}+\underline{q}_{s} \cdot \underline{\dot{\varepsilon}}^{p s} \geqslant 0
$$

\subsection{Summary}

Table 1 summarizes the internal variables, thermodynamic forces and threshold functions for the four nonlinear mechanisms taken into account by the model.

The constitutive model depends on 18 parameters (some of them need to be defined both in $x$ and $y$ directions, resulting in 21 numerical values).

The first 5 parameters ( 6 numerical values) presented in Table 2 define the elastic behavior and they correspond to the section geometry and the elastic mechanical parameters of concrete and steel reinforcement bars.

The remaining 13 parameters ( 15 numerical values) govern the nonlinear behavior of the model and are presented in Table 3.

Most of these parameters are of common practice in civil engineering, since they concern the geometrical characteristics of the section or basic steel and concrete material properties. The rest of the parameters have to be estimated with formulas

\begin{tabular}{|c|c|c|c|c|c|c|}
\hline Mechanisms & Internal variables & & & Thermodynamic forces & & Threshold functions \\
\hline Concrete damage & Damage variable & $d$ & & Energy release rate & $Y$ & $f_{d}(Y)$ \\
\hline Concrete cracking & Crack displacement & $\begin{array}{l}w_{n} \\
w_{t}\end{array}$ & $\underline{w} \equiv\left(\begin{array}{l}w_{n} \\
w_{t}\end{array}\right)$ & Macro concrete stress at cracks & $\underline{q_{r}}$ & $\begin{array}{l}f_{g n}\left(q_{r, n} ; w_{n} ; w_{n}^{\max }\right) \\
f_{g t}\left(q_{r, t} ; w_{t}\right)\end{array}$ \\
\hline Bond-slip stress & Inelastic steel-concrete slip & $\begin{array}{l}v_{x}^{p} \\
v_{y}^{p}\end{array}$ & $\underline{v}^{p} \equiv\left(\begin{array}{c}v_{x}^{p} \\
v_{y}^{p}\end{array}\right)$ & Macro bond-slip stress & $\underline{q} v$ & $\begin{array}{l}f_{v x}\left(q_{v, x}\right) \\
f_{v y}\left(q_{v y}\right)\end{array}$ \\
\hline Steel yielding & Steel plastic strain & $\begin{array}{l}\varepsilon_{x}^{p s} \\
\varepsilon_{y}^{p s}\end{array}$ & $\underline{\varepsilon}^{p s} \equiv\left(\begin{array}{c}\varepsilon_{x}^{p s} \\
\varepsilon_{y}^{p s}\end{array}\right)$ & Macro steel stress & $\underline{q}_{s}$ & $\begin{array}{l}f_{s x}\left(q_{s, x} ; \underline{w}, \underline{v}^{p}\right) \\
f_{s y}\left(q_{s, y} ; \underline{w}, \underline{v}^{p}\right)\end{array}$ \\
\hline
\end{tabular}
existing in the literature or with the indications given in Appendix D.

\section{Numerical application and comparison with experimental tests}

The proposed global RC panel constitutive model has been implemented in the DKTG plate finite elements in Code_Aster FE software. The fully implicit integration scheme of Code_Aster is used, including the computation of a tangent stiffness

Table 1

Summary of the model internal variables, thermodynamic forces and threshold functions. 
Model parameters for the linear elastic behavior.

\begin{tabular}{llll}
\hline Section geometry parameters & $h$ & {$[\mathrm{~m}]$} & Plate thickness \\
& $A_{s \alpha}$ & {$\left[\mathrm{m}^{2} / \mathrm{m}\right]$} & Reinforcement section in the $\alpha$ direction per unit of length \\
Material elastic properties & $E_{c}$ & {$[\mathrm{~Pa}]$} & Initial (undamaged) concrete Young's modulus \\
& $v_{c}$ & {$[-]$} & Concrete Poisson's ratio \\
& $E_{s}$ & {$[\mathrm{~Pa}]$} & Steel Young's modulus \\
\hline
\end{tabular}

Table 3

Model parameters for the nonlinear behavior.

\begin{tabular}{llll}
\hline Concrete tensile behavior & $f_{c t}$ & {$[\mathrm{~Pa}]$} & Concrete tensile strength \\
& $G_{f}$ & {$\left[\mathrm{~J} / \mathrm{m}^{2}\right]$} & Concrete fracture energy \\
& $\alpha_{u}$ & {$[-]$} & Ratio of $E_{c}$ accounting for the unload slope \\
& $\alpha_{r}$ & {$[-]$} & Ratio of reclosing crack opening over maximum historical crack opening \\
& $s_{r \alpha}$ & {$[\mathrm{m}]$} & Theoretical average crack spacing in the $\alpha$ direction \\
Concrete damage parameters & $k_{o}$ & {$[\mathrm{~Pa}]$} & Constant threshold of the energy release rate \\
& $\gamma_{d}$ & {$[-]$} & Fraction of $E_{c}$ corresponding to the asymptotic fully damaged concrete slope \\
Aggregate interlock & $T_{o}$ & {$[\mathrm{~Pa}]$} & Initial crack shear resistance \\
& $T_{1}$ & {$[\mathrm{~Pa} / \mathrm{m}]$} & Stiffness of the aggregate interlock \\
Steel yielding & $f_{s y}$ & {$[\mathrm{~Pa}]$} & Steel yielding stress \\
Bond stresses and tension stiffening & $K_{l}$ & {$[\mathrm{~Pa} / \mathrm{m}]$} & Initial local bond-slip tangent stiffness \\
& $k_{t}$ & {$[-]$} & Maximum average tension stiffening coefficient in the concrete \\
& $\phi_{\alpha}$ & {$[\mathrm{m}]$} & Diameter of reinforcement bars in the $\alpha$ direction \\
\hline
\end{tabular}

matrix, in order to ensure the fulfillment of the constitutive equations, namely the consistency of the internal variables evolutions on each time step. This numerical choice takes full advantage of the theoretical framework adopted before.

It is noted that the numerical implementation of the developed stress resultant model does not suffer in general from mesh dependency due to strain localization. The retained global modeling approach representing at the same time concrete and steel reinforcement has led to the definition of the crack spacing $s_{r}$ as a parameter which is determined at cracking onset by an analytical expression (see Section 3.2) and then remains fixed, affecting the threshold function of the concrete bridging stress (5-15) and regularizing the energy dissipation surface density due to crack opening $D_{\mathrm{r}}$ over its length:

$$
D_{r}=\int_{0}^{+\infty} q_{r, n} d w_{n}=\int_{0}^{+\infty} \frac{\rho_{c} h}{s_{r}} g_{n} d w_{n}=\frac{\rho_{c} h}{s_{r}} \int_{0}^{+\infty} G_{n}\left(w_{n}\right) d w_{n}=\frac{\rho_{c} h}{s_{r}} G_{f}
$$

For example, an element of dimension $m s_{r}$ in the direction orthogonal to the crack (accounting for $m$ cracks) dissipates $m$ times the energy dissipated by a single crack (fracture energy $G_{f}$ multiplied by the concrete section $\rho_{c} h$ ). However, if the element has a dimension (in the direction orthogonal to crack) smaller than the crack spacing and the model presents a global softening behavior (e.g. because of a low reinforcement ratio), it will suffer from mesh dependency, since the total dissipated energy will be less than the energy dissipated by one crack. Nevertheless, this problem is rare because:

- The common finite element size in a global approach is typically several times larger than the crack spacing in a RC plate, which is of the order of $10 \mathrm{~cm}$.

- The proposed model represents the RC composite mechanical behavior that usually shows a positive tangent stiffness, because the stiffness of the steel reinforcement before yielding often compensates a possible softening behavior of the concrete part (due to damage evolution or crack opening in tension), see e.g. the force-strain curves of Fig. 6-2. Therefore, no strain localization in a unique element occurs and the dissipated energy is correctly estimated by considering the total cracked surface (formed by many elements).

\subsection{Monotonic pure tension tests}

The model is first validated by comparison with the uniaxial pure tension tests N10-10, N10-14 and N10-20 on RC tie beams with steel reinforcing bars only in the longitudinal direction of Farra and Jaccoud [11]. In order to show the global modeling performance of the developed model, only one shell finite element is used for representing a RC beam, as shown in Fig. 6-1. The length $L$ and width $b$ of the beams are the dimensions of the element while the height is the model parameter $h$.

The three tests concern beams with length $L=1.15 \mathrm{~m}$ length and $100 \times 100 \mathrm{~mm}^{2}$ section $(b=h=0.1 \mathrm{~m})$ made by a concrete characterized by the experimentally measured Young's modulus $E_{c}=29.9 \mathrm{GPa}$, tensile strength $f_{c t}=2.13 \mathrm{MPa}$ and compressive strength $f_{c}=29.9 \mathrm{MPa}$.

The beams are only reinforced in the $x$ direction with one steel reinforcement bar located at the center of the section and their characteristics are summarized in Table 4. 


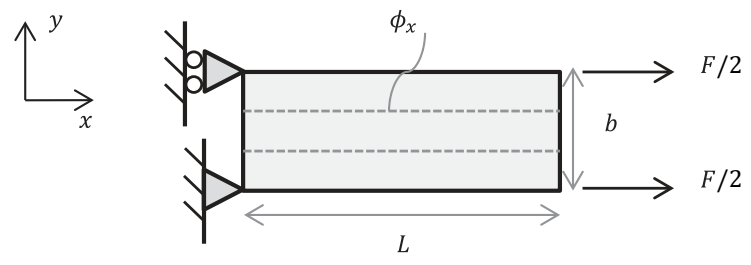

Fig. 6-1. FE model and boundary conditions for an RC beam of length $L$ and width $b$ submitted to a tension and/or compression load $F$.

Table 4

Steel reinforcement bar characteristics for N10-10, N10-14 and N10-20 tests of Farra and Jaccoud [11].

\begin{tabular}{lllll}
\hline Test & Bar diameter $\phi_{x}[\mathrm{~mm}]$ & Reinforcement ratio [\%] & Steel Young's Modulus $E_{s}[\mathrm{GPa}]$ \\
\hline N10-10 & 10 & 0.79 & 202 & 590 \\
N10-14 & 14 & 1.54 & 198 & 569 \\
N10-20 & 20 & 3.14 & 201 & 530 \\
\hline
\end{tabular}

The theoretical crack spacing parameter $s_{r x}$ used for the model is assumed equal to the average spacing measured in the tests: $s_{r x}=220 \mathrm{~mm}$ for $\mathrm{N} 10-10, s_{r x}=173 \mathrm{~mm}$ for $\mathrm{N} 10-14$ and $s_{r x}=122 \mathrm{~mm}$ for $\mathrm{N} 10-20$. Thanks to this choice, modeling errors coming from the calculation of crack spacing by an empirical formula like (D-2) are avoided.

Concerning the concrete tensile strength, the test report indicates that the experimental observed value for $f_{c t}$ for each of the three tests is $89 \%, 81 \%$ and $63 \%$ of the nominal value of $2.13 \mathrm{MPa}$; these are the retained values for the computations. Two phenomena cause the $f_{c t}$ decrease: (i) the size effect between the tensile strength test sample and the RC beams and (ii) concrete shrinkage, which (as suggested by the authors of the test) is more restrained for the case of large reinforcement bars, leading to a significant initial tension state in concrete. Then, the theoretical fracture energy $G_{f}=135 \mathrm{~J} / \mathrm{m}^{2}$ given by (D-1) is also decreased for each beam in order to keep the theoretical value of the post-peak slope $f_{c t}^{2} /\left(2 G_{f}\right)$ of the envelope bridging stress law (5-17).

In accordance to Appendix D, the local bond-slip stiffness is estimated to $K_{l}=10^{11} \mathrm{~Pa} / \mathrm{m}$ and the tension stiffening coefficient is set to $k_{t}=0.6$ according to MC10, which is much less than the limit defined by (5-36) for the thermodynamic admissibility (equal to $2.5,5.3$ and 14.2 respectively for the three tests).

In this test, numerical results are not affected by (i) concrete Poisson's ratio $v_{c}$ (uniaxial stress state), (ii) steel properties in the $y$ direction $\left(A_{s y}=0 \mathrm{~cm}^{2} / \mathrm{m}\right.$ ), (iii) unloading and reloading bridging stress parameters $\alpha_{u}$ and $\alpha_{r}$ (no cycling load considered), (iv) aggregate interlock parameters $T_{o}$ and $T_{1}$ (no tangential displacement in uniaxial test) and (v) damage parameters $\gamma_{d}$ and $k_{o}$ (since from recommendations in Appendix D, the energy release rate threshold has to be chosen in order to have damage onset at $\sigma_{d} \in\left[f_{c} / 4, f_{c} / 2\right]$, so concrete threshold stress values never reached in a pure tension test).

In the experimental campaign, N10-14 test has been repeated twice and N10-10 and N10-20 tests have been repeated three times. The comparison between experimental and numerical results is presented in Fig. 6-2, showing a satisfactory agreement for both for stress and crack opening values. Actually, there is a slight underestimation of individual crack openings and an almost perfect fit of the total crack opening (the sum of the openings of all the existing cracks in the element/ beam). This is a consequence of the modeling choice made in Section 3.2, consisting in avoiding the representation of the crack formation phase. In the numerical computation, the element reaches the stabilized crack stage immediately after the onset of the first crack and the crack spacings stay constant and equal to the final average crack spacing (relatively small), while in the experimental tests the crack pattern converges to the final stabilized crack state after a transient phase characterized by a few largely-spaced cracks. Therefore, in the experiment, at the onset of the first crack, the crack spacing is much larger than the average final one and thus the measured crack openings are also larger. Fortunately, this source of error on crack opening is limited because (i) the crack formation phase is short since few cracks form within a finite element with the typical modeling size $(\approx 10 \mathrm{~cm}-50 \mathrm{~cm})$ and (ii) it affects only the first tension cycle in cyclic loadings.

\subsection{Uniaxial cyclic tension-compression test}

The experimental test QJ5TC realized by Benmansour [2] is retained to validate the model when submitted to the pseudostatic uniaxial cyclic tension-compression load history of Fig. 6-3, carried out at low strain velocity $\left(10^{-3} / \mathrm{min}\right)$. Using the same FE modeling and notation of Fig. 6-1, the test consists in a RC beam of $L=0.7 \mathrm{~m}$ and of $150 \times 150 \mathrm{~mm}^{2}$ section $\left(b=h=0.15 \mathrm{~m}\right.$ ), reinforced with 4 ribbed steel bars of $\phi_{x}=14 \mathrm{~mm}$ diameter with a Young's modulus $E_{s}=195 \mathrm{GPa}$ and a yield stress of $f_{s y}=610 \mathrm{MPa}$. Concrete is characterized by its Young's modulus $E_{c}=28.5 \mathrm{GPa}$, tensile strength $f_{c t}=2.94 \mathrm{MPa}$ and compressive strength $f_{c}=25 \mathrm{MPa}$. The effective value for $f_{c t}$ due to the size effect is estimated to be 
N10-10
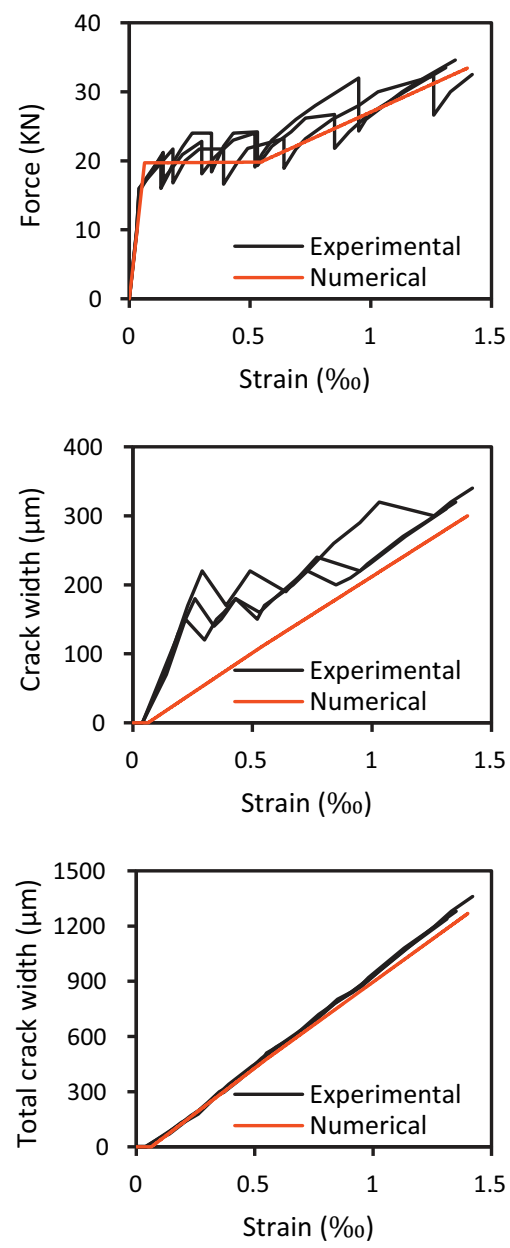

$\mathrm{N} 10-14$
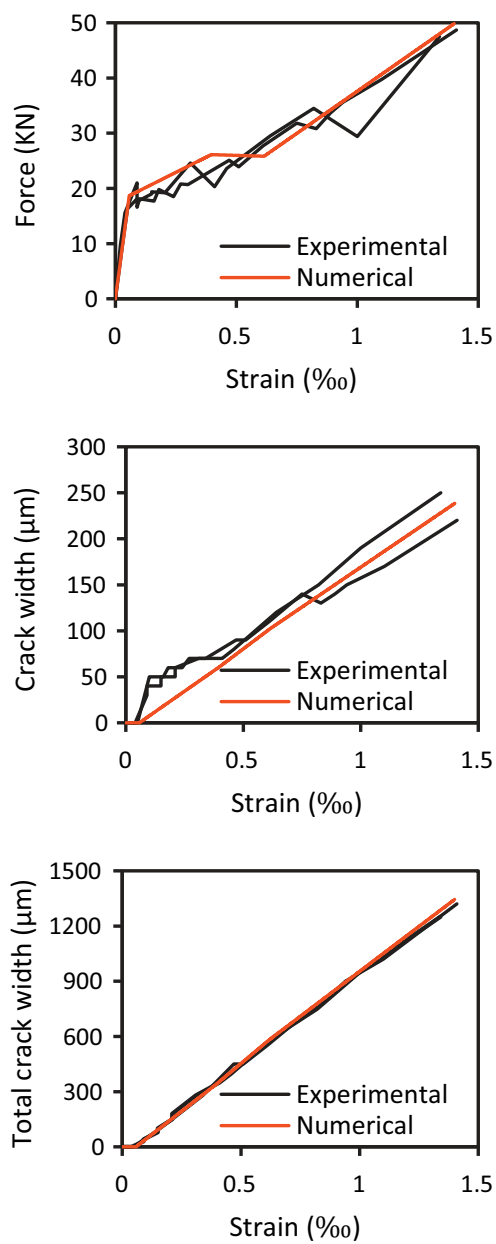

N10-20
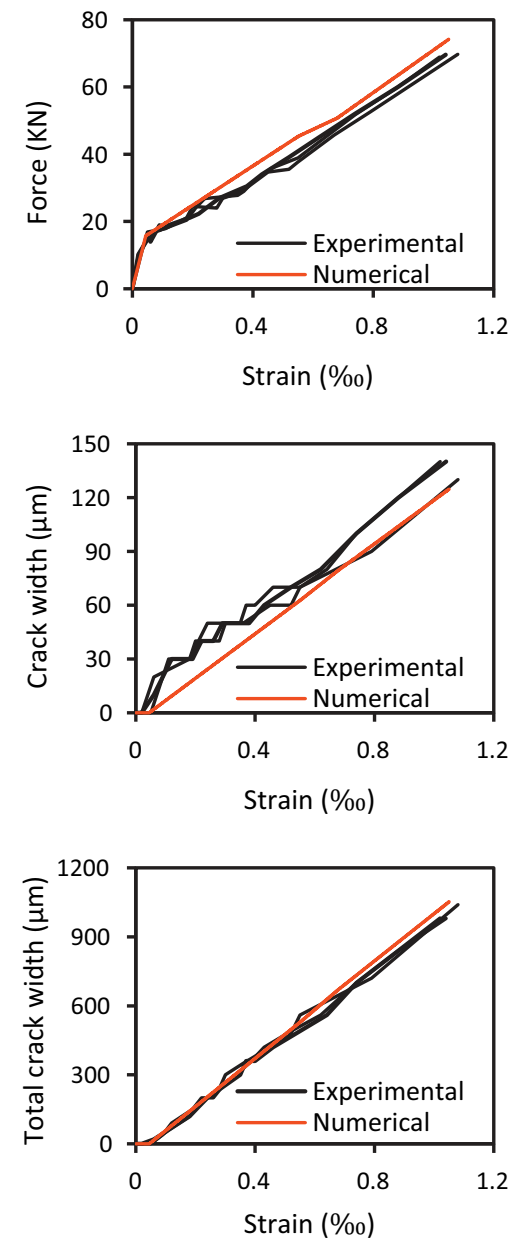

Fig. 6-2. Comparison between numerical and experimental monotonic test results for N10-10, N10-14 and N10-20 of Farra and Jaccoud [11].

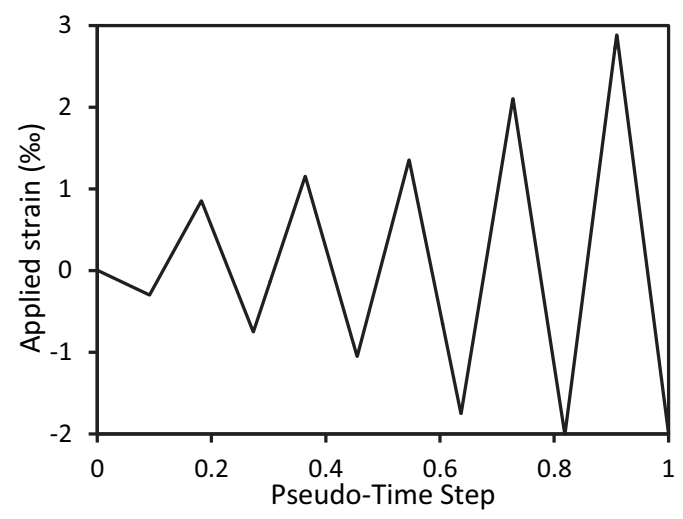

Fig. 6-3. Applied strain history. 
$86 \%$ of the value measured on a cylindrical sample of $160 \mathrm{~mm}$ diameter and $320 \mathrm{~mm}$ height, according to the expression given by Rossi et al. [26].

Following the recommendations of Appendix D, the theoretical average crack spacing is estimated to $s_{r}=87 \mathrm{~mm}(\mathrm{D}-2)$; the theoretical concrete fracture energy of $G_{f}=130 \mathrm{~J} / \mathrm{m}^{2}$ (D-1) is reduced with the square of the concrete tensile strength reduction due to size effect, so a value of $G_{f}=96.4 \mathrm{~J} / \mathrm{m}^{2}$ is retained; the values of the parameters of cyclic bridging stress are retained in their low limit $\alpha_{r}=\alpha_{u}=0.01$ (no tangential-to-crack effects appear in a uniaxial test); a damage threshold of $k_{0}=1920 \mathrm{~Pa}$ is adopted to set the damage onset at $f_{c} / 2$ with a concrete damage evolution slope of $\gamma_{d}=0.3$; bond-slip parameters are chosen as $k_{t}=0.6$ (less than the limit value of $k_{t} \leqslant 6.8$ established by (5-36)) and $K_{l}=10^{11} \mathrm{~Pa} / \mathrm{m}$, the same values than in the previous uniaxial pure tension test.

In this test, numerical results are not affected by (i) concrete Poisson's ratio $v_{c}$ (uniaxial stress state), (ii) steel properties in the $y$ direction $\left(A_{s y}=0 \mathrm{~cm}^{2} / \mathrm{m}\right)$ and (iii) aggregate interlock parameters $T_{o}$ and $T_{1}$ (no tangential displacement in uniaxial test).

Fig. 6-4 shows that there is a good agreement between experimental and numerical stress-strain curves in tension, which implies that concrete cracking (including crack opening-reclosing) and bond-slip phenomena are well modeled. However, the agreement in compression is less satisfactory because damage is not sufficient to accurately represent the hysteretic loops in compression. Fig. 6-6 shows the evolution of the dissipated energy in the beam and the contribution of each of the considered non-linear phenomena, depending on the evolution of the internal variables of the model, as expressed in (5-37). The evolution of the state variables, graphically represented in Fig. 6-5, shows that the crack opening-reclosing follows the applied tension cycles, the inelastic slip and the plastic steel strain at cracks are only activated at the last tension cycles and the damage variable only evolves during compression.

\subsection{Application to the non-reversing cyclic shear test on RC wall 3 of CEOS.fr program}

The considered experimental test is the RC wall number 3 designed and tested in the framework of the French national research project CEOS.fr (Comportement et Evaluation des Ouvrages Spéciaux. Fissuration - Retrait / Behavior and Assessment of Special Structures. Cracking - Shrinkage), already partially presented in Section 3.2.

\subsubsection{Experimental test description}

The RC wall number 3 of the CEOS.fr program represents a standard RC wall used in nuclear facilities with an assumed geometrical scale factor of $1 / 3$. The retained dimensions of this mock-up are $4200 \mathrm{~mm}$ of length, $1050 \mathrm{~mm}$ of height and $150 \mathrm{~mm}$ of thickness. Cracking due to bending is prevented by reinforcing the extremities with vertical rebars of $25 \mathrm{~mm}$ and $32 \mathrm{~mm}$ diameter and by a height/length ratio of $1 / 4$ which ensures a low slenderness of the wall. The redistribution of the shear effort in the wall is guaranteed by two horizontal concrete beams with high reinforcement ratio connected to its upper and bottom edges.

In order to extend the crack formation phase before complete failure, the non-brittleness of the wall is assured by a $1.05 \%$ reinforcement ratio, in both "top" and "bottom" layers. Reinforcement is characterized by a bar diameter of $\phi_{\alpha}=10 \mathrm{~mm}$ spaced by $100 \mathrm{~mm}$, so the steel section is $A_{s \alpha}=15.78 \mathrm{~mm}^{2} / \mathrm{m}$. Concrete cover is $10 \mathrm{~mm}$ for the horizontal rebars and $20 \mathrm{~mm}$ for the vertical ones. The measured steel mechanical properties are: Young's modulus $E_{s}=200 \mathrm{GPa}$, mass density $7850 \mathrm{~kg} / \mathrm{m}^{3}$ and yield limit $f_{s y}=555 \mathrm{MPa}$. Concrete experimental properties are: Young's modulus $E_{c}=27.4 \mathrm{GPa}$, Poisson's

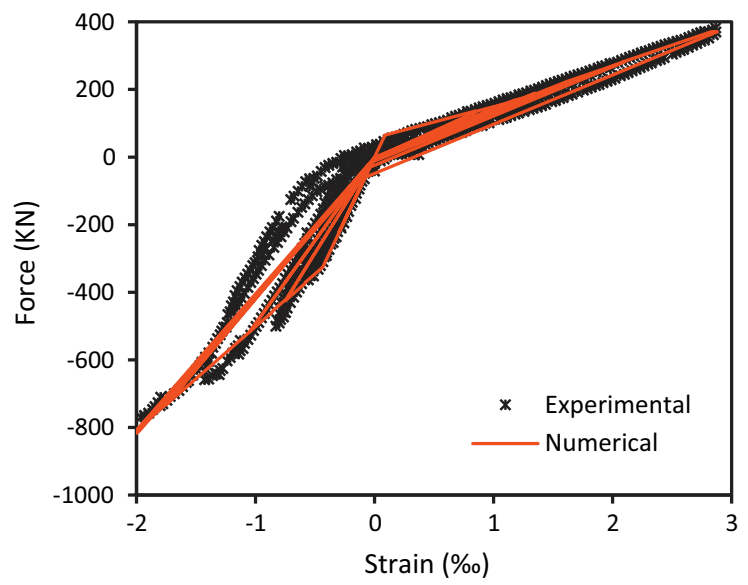

Fig. 6-4. Force-strain comparison between numerical and experimental results for the tension-compression test QJ5TC of Benmansour [2]. 


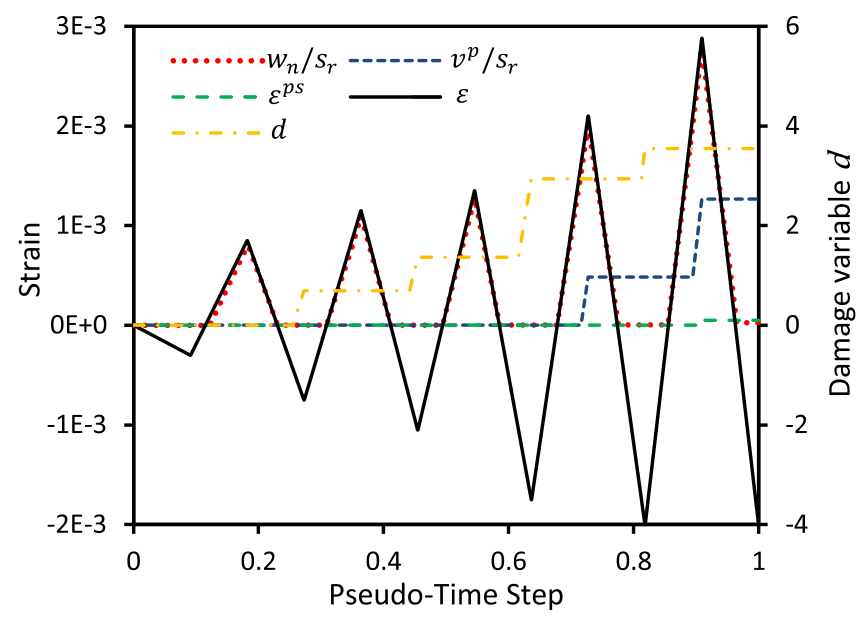

Fig. 6-5. Numerical evolution of the state variables.

ratio $v_{c}=0.13$, mass density $2200 \mathrm{~kg} / \mathrm{m}^{3}$, compression strength $f_{c}=40.0 \mathrm{MPa}$, tensile strength $f_{c t}=3.5 \mathrm{MPa}$ and fracture energy $G_{f}=158 \mathrm{~J} / \mathrm{m}^{2}$.

Fig. 6-7 shows how RC wall forms with the steel frame a self-equilibrated system: at the left-bottom side, the horizontal beam distributes the stress transferred through the wall to the steel frame, where a mortar joint is used to diffuse the horizontal reaction over the height of the beam. The vertical displacements of the wall are restrained by two couples of Dywidag bars with a $1000 \mathrm{kN}$ pre-stress load. The instrumentation installed on the wall is complex, here the LVDT (Linear Voltage Differential Transformers) C5, C6, C7, C9 and C10 sensors are highlighted. The first three sensors measure the evolution of three crack widths, corresponding to the crack pattern formed in the wall characterized by an average crack orientation of $\theta_{r}=28^{\circ}$. The difference of displacements between the last two sensors defines the global force-displacement curve which is used for the numerical comparison in Fig. 6-10, when the cyclic (without inversion of the force sign) load history of Fig. 6-8 is applied.

\subsubsection{Modeling of the RC wall}

The RC wall is modeled by quadrangular plate finite elements. As seen in Fig. 6-9, realistic boundary conditions have been represented including the steel frame, which is modeled with beam elements.

Concrete size effect on tensile strength is complex to be estimated in a structure with a non-uniform stress state. Taking into account the observed and calculated values for the size effect in the RC beams (with smaller dimensions) of the previous section, the experimental $f_{c t}$ value is reduced by $2 / 3$ and a value of $f_{c t}=2.33 \mathrm{MPa}$ is retained. As previously stated, the value of the fracture energy has to be reduced with the square of the $f_{c t}$ reduction in order to preserve the theoretical post-peak slope of the bridging stress curve and thus, a value of $G_{f}=71.1 \mathrm{~J} / \mathrm{m}^{2}$ is used in computations.

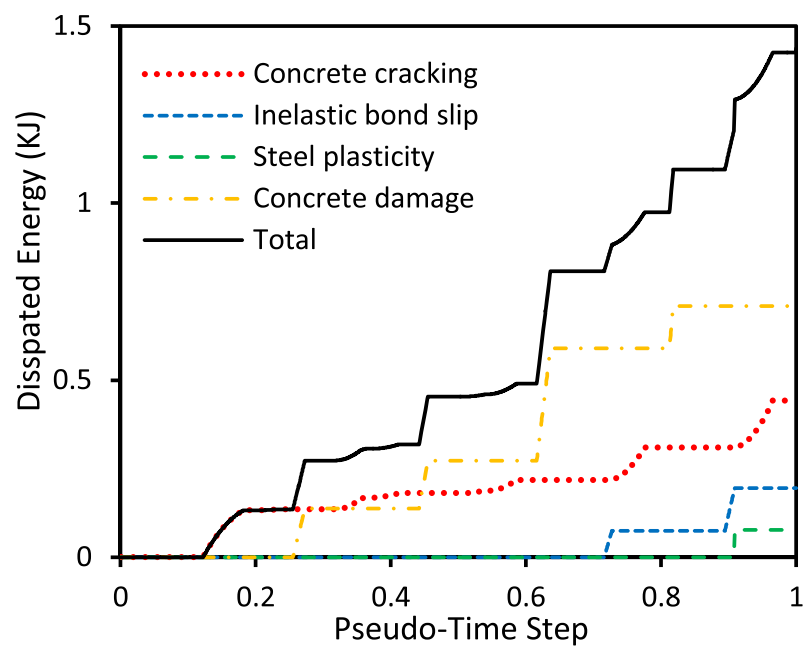

Fig. 6-6. Numerical energy dissipation for the different non-linear phenomena. 


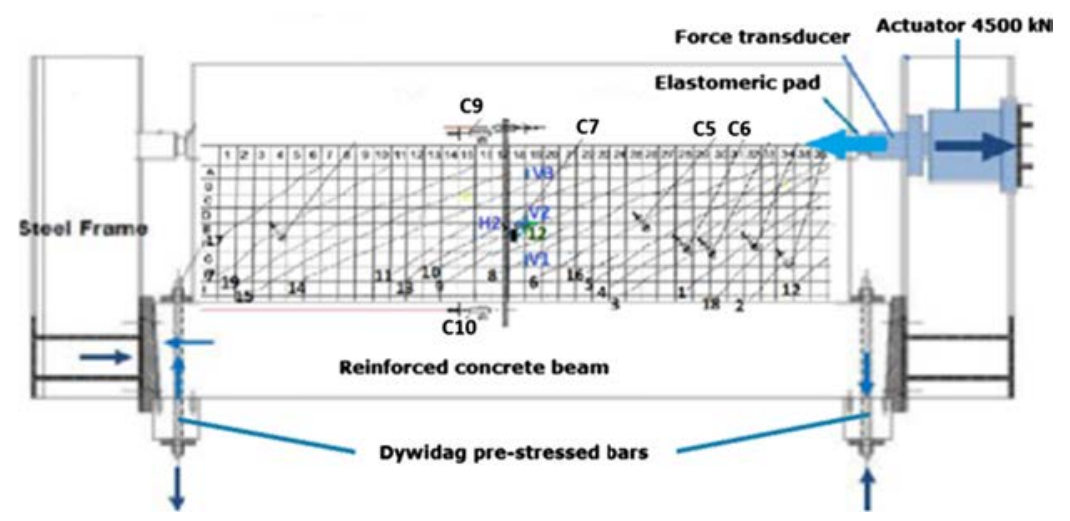

Fig. 6-7. Scheme of the RC wall of CEOS.fr with the steel frame structure and its instrumentation.

With the recommendations of Appendix D, concrete damage is supposed to appear in compression at $\sigma_{d}=f_{c} / 4$ and the asymptotic damage slope is set to $\gamma_{d}=0.3$, so (D-3) gives a damage threshold of $k_{o}=1277 \mathrm{~Pa}$. The theoretical average crack spacings are calculated with (D-2), obtaining $s_{r x}=124 \mathrm{~mm}$ and $s_{r y}=138 \mathrm{~mm}$, which differ due to the different cover in each direction. Between cracks, a relatively low tension stiffening effect is supposed to be developed in reason of the cyclic loading: $k_{t}=0.2$, which satisfies the limit value $k_{t} \leqslant 3.4$ established by (5-36). As in the previous tests, the local bond-slip stiffness is estimated to $K_{l}=10^{11} \mathrm{~Pa} / \mathrm{m}$. In reason of the cyclic loading and the evolution at the same time of normal $w_{n}$ and tangential $w_{t}$ crack displacements, the values of the cyclic bridging stress parameters $\alpha_{r}=0.02, \alpha_{u}=0.05$ are retained. As moderate crack openings are expected, aggregate interlock parameters are estimated to $T_{o}=0.05 \mathrm{MPa}$ and $T_{1}=10 \mathrm{GPa} / \mathrm{m}$.

\subsubsection{Comparison between numerical and experimental results}

The experimental loading history of Fig. 6-8 is applied on the FE model of the RC wall. Fig. 6-10 shows the global forcedisplacement curve comparison between the experimental and the numerical results. It is noted that the envelope curve, the permanent displacements (related to the remaining crack openings, governed by the crack reclosing ratio $\alpha_{r}$ ) and the hysteretic loops (partially governed when unloading by $\alpha_{u}$ parameter) are well represented, especially for the first load cycles.

The numerical and experimental crack pattern evolutions in the RC wall are compared in Fig. 6-11 for three different load levels: 1500, 2400 and $3600 \mathrm{KN}$, the last one corresponding to the experimentally observed stabilization of crack pattern. The numerical crack pattern is represented by the zones where the crack opening internal variable is greater than 0 (color different from blue). The comparison shows that the constitutive model is able to predict that the first cracks appear in the lower right corner of the wall and then cracking propagates gradually to the left. The experimental average crack orientation of $\theta_{\mathrm{r} 1} \approx 28^{\circ}$ is found in the generated crack pattern in the RC wall far from the edges.

Fig. 6-12 presents a local analysis concerning the measured crack openings in LVDT sensors C5, C6 and C7, located at positions shown in Fig. 6-7. The experimental crack opening for each sensor at each peak load cycle (see load history of Fig. 6-8) is compared with the average internal variable $w_{n}$ in the finite element in which each sensor is located. It can be noted that in the three cases the crack onset is well represented (related to the crack pattern evolution analyzed in Fig. 6-11). The crack onset and opening evolution with the increasing load is well fitted for all sensors C5, C6 and C7. One part of the slight observed difference is originated by the punctual measures of crack opening values made by LVDT sensors, which are in some cases not too much representative of the average crack opening in a zone (or element in this case). As shown by

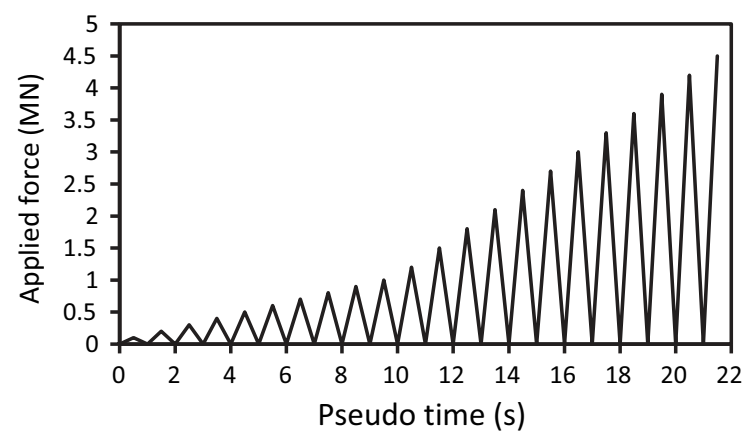

Fig. 6-8. Cyclic non-reversing load history applied to the RC wall. 


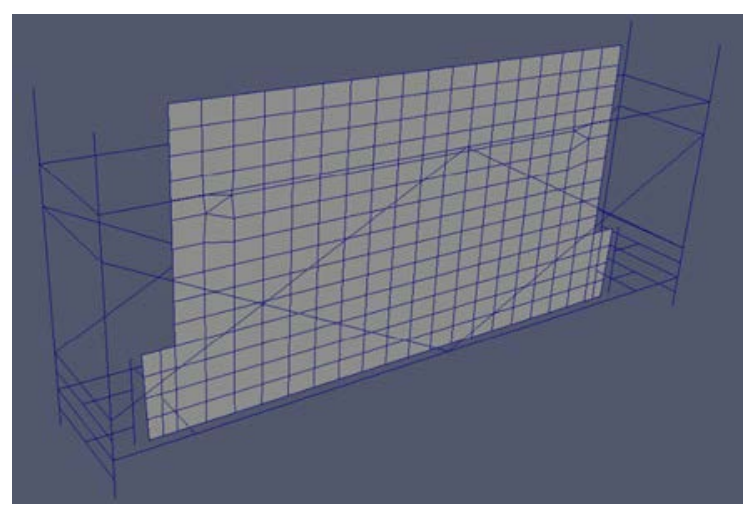

Fig. 6-9. FE model of the RC wall 3 of CEOS.fr.

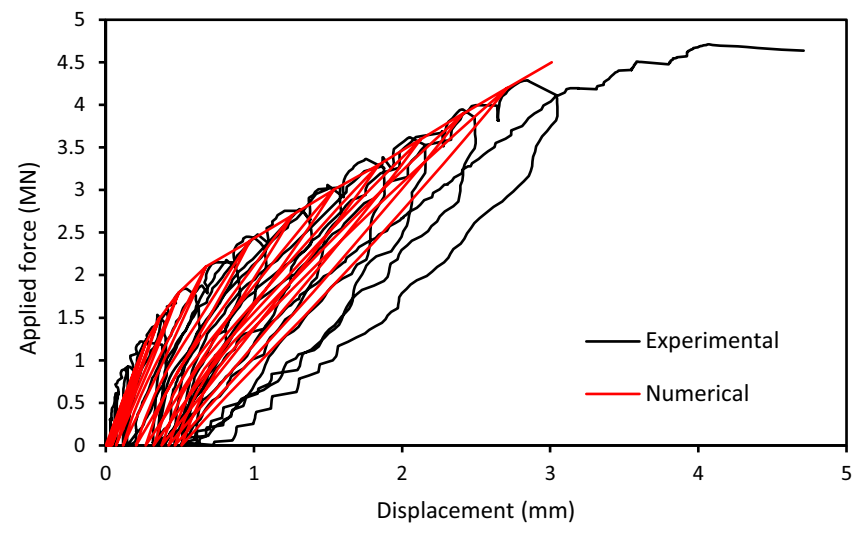

Fig. 6-10. Experimental vs. numerical global force-displacement curve.

the digital image correlation analysis on the wall performed by Ruocci et al. [27], crack opening is not constant along cracks, with important variations at the scale of $\mathrm{mm}$ and $\mathrm{cm}$ because of the roughness of the crack surface.

The global in-plane stiffness reduction of the RC panel at a time $T$ can be estimated by the scalar value $K_{r d}(T)$ :

$$
K_{r d}(T)=1-\sqrt[3]{\prod_{i=1}^{3} \frac{\mathbb{A}_{i i i i}(d(T))}{\mathbb{A}_{i i i i}(0)}}
$$

The distribution of this estimator in the entire wall at the end of the test is shown in Fig. 6-13. The maximum stiffness reduction $\left(K_{r d} \approx 0.6\right)$ is obtained at the force application point (upper right side of the wall) and at the mortar joint that transfers the stress to the steel frame at the left bottom side, according to the scheme of the force transfers shown in Fig. 6-7. Stiffness reduction is less important in the center of the wall (roughly 0.3 ) because of the diffusion of compressive stresses in this zone.
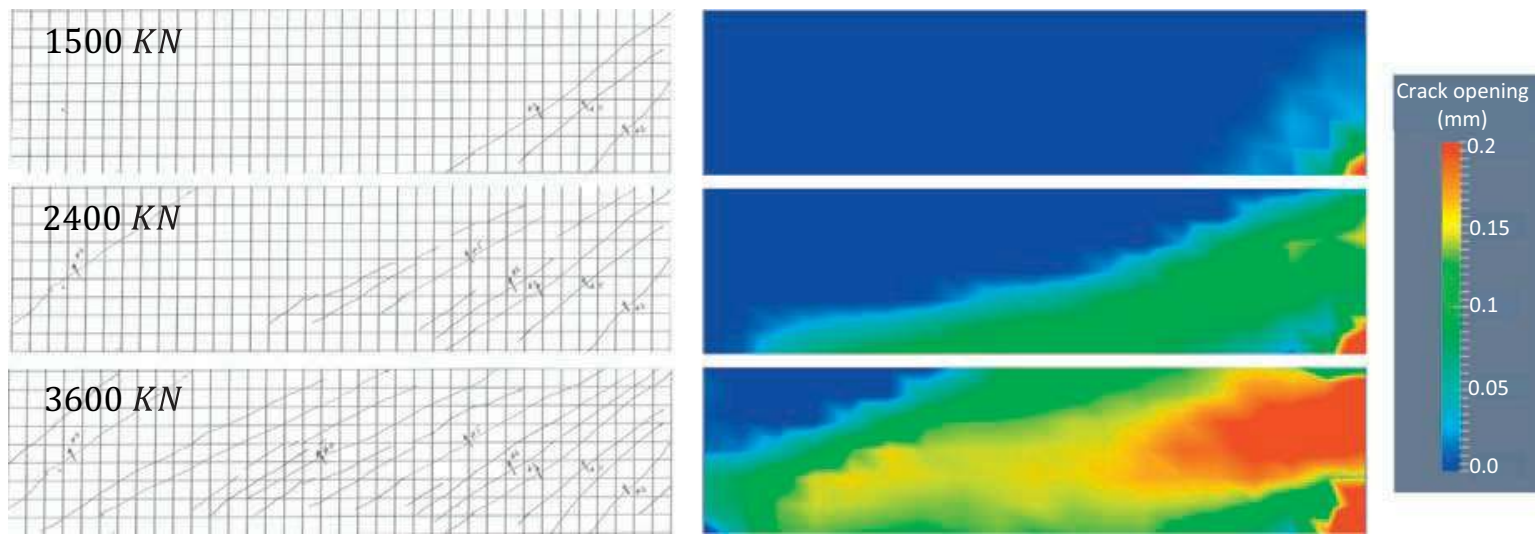

Fig. 6-11. Crack extension comparison between experimental and numerical results for 1500, 2400 and $3600 \mathrm{KN}$ load steps. 

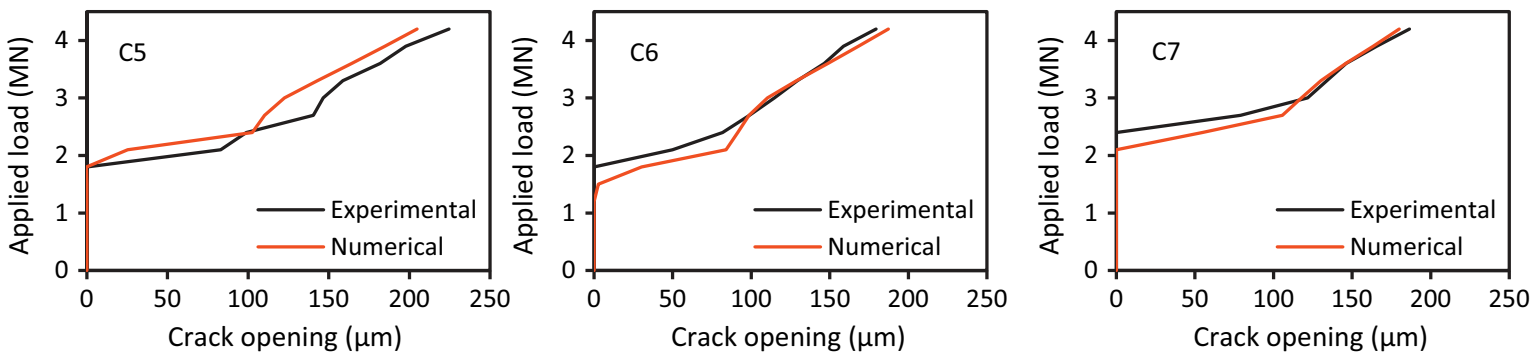

Fig. 6-12. Comparison of numerical and experimental crack openings at sensors C5, C6 and C7.
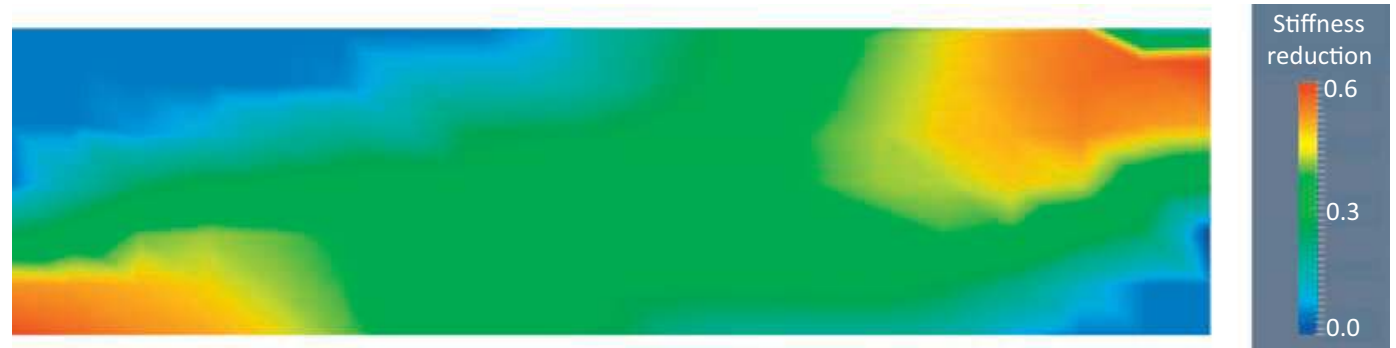

Fig. 6-13. Numerical stiffness reduction at the end of CEOS.fr RC wall 3 test.

Finally, it is noted that no steel reinforcement yielding has been obtained during the numerical simulation (homogeneous $\varepsilon_{\alpha}^{p s}=0$ in the entire RC wall), which is in accordance with the experimental observation.

\section{Conclusion}

A novel stress resultant nonlinear constitutive model for RC panels submitted to membrane cyclic loadings has been proposed in this paper. The model takes into account four important local scale nonlinear physical phenomena in RC structures: (i) concrete damage due to micro-cracking in compression, resulting in concrete stiffness degradation, (ii) concrete cracking, considering displacement and stress transfer in both normal and tangential directions with respect to the crack, (iii) bond stresses between steel bars and concrete due to a relative slip and (iv) steel reinforcement yielding at crack crossings. In order to describe these phenomena for a robust and efficient FE numerical implementation, an analytical multi-scale analysis has been realized within a RVE defined by the crack pattern geometry, taking full advantage of equilibrium statements to obtain the stress resultant expression. Furthermore, the model equations have been developed in the framework of the Thermodynamics of Irreversible Processes and the Generalized Standard Material Theory, in order to ensure the robustness of the numerical integration and the appropriate energy dissipation description for all possible loading situations for the FE analysis of an RC structure. The comparisons of the proposed model with experimental uniaxial pure tension and tensioncompression tests on RC beams and a cyclic (non-reversing) shear test on a RC wall show good agreement for both stress-strain curves and crack opening evolution. Moreover, the model allows analyzing the relative contribution of each dissipative phenomenon throughout the loading history. Future developments are devoted to the extension of this constitutive model to (i) the possible onset of a second crack family (which differs from the first one in its crack orientation, spacing and displacement) and (ii) to the combined out-of-plane bending plus membrane loads. Also, more sophisticated laws for nonlinear phenomena will be analyzed, especially concerning aggregate interlock.

\section{Acknowledgements}

The authors wish to thank EGIS Industries, Ecole Centrale Nantes, Electricité de France R\&D Division, and Association Nationale de la Recherche et de la Technologie for their financial and material support for conducting these researches, and also Prof. P. Bisch and Prof. L. Davenne for their valuable comments and suggestions. The financial support from Agence Nationale de la Recherche under the program "Future Investments", via the SINAPS@ project, reference No. ANR-11-RSNR002, is also gratefully acknowledged. 


\section{Appendix A}

The steel-concrete slip is an unknown function $s=s(\xi)$ which has to be determined. The equilibrium on an RC beam subjected to tension gives the following differential equation:

$$
s^{\prime \prime}(\xi)-s_{r}^{2} \frac{\left(1+\frac{\rho_{s} E_{s}}{\rho_{c} E_{c}}\right)}{\phi E_{s}} \tau(s(\xi))=0
$$

with $\tau(s(\xi))=K_{l} s(\xi)$, see (3-5). The retained boundary conditions are no slip at the mid-section between two cracks and a slip at crack equal to $v$ (equal to $w_{n} / 2$ ):

$$
s(0)=0 s( \pm 1)=v
$$

The solution of the previous differential equation is given by:

$$
s(\xi)=\frac{\sinh \left(\sqrt{C K_{l}} \cdot \xi\right)}{\sinh \left(\sqrt{C K_{l}}\right)} v
$$

where

$$
C=s_{r}^{2} \frac{\left(1+\frac{\rho_{s} E_{s}}{\rho_{c} E_{c}}\right)}{\phi E_{s}}
$$

With the obtained expression for the slip function $s(\xi)$, the average tension stiffening effect (4-20) can be calculated:

$$
\tau^{0}=\frac{1}{2} \int_{-1}^{1} \int_{|\xi|}^{1}-K_{l} \frac{\sinh \left(\sqrt{C K_{l}} \cdot z\right)}{\sinh \left(\sqrt{C K_{l}}\right)} v d z d \xi=\frac{1}{C}\left(1-\frac{\sqrt{C K_{l}}}{\tanh \left(\sqrt{C K_{l}}\right)}\right) v
$$

\section{Appendix B}

In this appendix, it is proved that condition (5-36), together with the conditions given by the threshold functions (5-24) and (5-29), is sufficient to proof that (5-35) holds for any load history:

$$
q_{s \alpha} \dot{\lambda}_{s \alpha} \operatorname{sign}\left(q_{s, \alpha}+s_{r, \alpha}\left(\boldsymbol{K}^{s} \cdot\left(\boldsymbol{M}^{v w} \cdot \underline{w}-\underline{v}^{p}\right)\right) \cdot \underline{e}_{\alpha}\right) \geqslant 0
$$

If $\dot{\lambda}_{s_{\alpha}}=0$, (B-1) is fulfilled.

If $\dot{\lambda}_{s_{\alpha}}>0$, (B-1) can be divided by $\dot{\lambda}_{s_{\alpha}}$ and the condition becomes:

$$
A \operatorname{sign}(A+B) \geqslant 0
$$

with the identification:

$$
A=q_{s \alpha} \quad B=s_{r, \alpha}\left(\boldsymbol{K}^{s} \cdot\left(\boldsymbol{M}^{v w} \cdot \underline{w}-\underline{v}^{p}\right)\right) \cdot \underline{e}_{\alpha}
$$

Condition (B-2) can be simplified as:

$$
A(A+B) \geqslant 0 \leftrightarrow A^{2}+A B \geqslant 0
$$

B.1. Case $A=0$ or $B=0$

$$
\begin{aligned}
& \text { If } A=0 \text { or } B=0,(B-4) \text { is fulfilled: } \\
& \qquad \begin{array}{l}
A=0 \rightarrow 0 \geqslant 0 \\
B=0 \rightarrow A^{2} \geqslant 0
\end{array}
\end{aligned}
$$

\section{B.2. Case $A B>0$}

If $A$ and $B$ have the same sign, then $A B>0$. Hence, (B-4) is the sum of two positive terms and it is fulfilled.

\section{B.3. Case $A B<0$}

The last possibility is that $A$ and $B$ have opposite signs and are both different from zero: $A B<0$. With this condition, (B-4) becomes: 


$$
A^{2}+A B \geqslant 0 \leftrightarrow A^{2} \geqslant-A B \leftrightarrow-\frac{A}{B} \geqslant 1 \leftrightarrow\left|\frac{A}{B}\right| \geqslant 1 \leftrightarrow \frac{|A|}{|B|} \geqslant 1 \leftrightarrow|A| \geqslant|B|
$$

The Kuhn-Tucker condition implies $f_{s \alpha}=0$ and the following condition is found using (5-29):

$$
|A+B|=\rho_{s \alpha} h f_{s y}
$$

If $|B| \leqslant \rho_{s \alpha} h f_{s y \alpha}$ :

$$
\left\{\begin{array} { l } 
{ | B | \leqslant \rho _ { s \alpha } h f _ { s y } } \\
{ | A + B | = \rho _ { s \alpha } h f _ { s y } }
\end{array} \rightarrow \left\{\begin{array}{l}
|B| \leqslant \rho_{s \alpha} h f_{s y} \\
|A|-|B|=\rho_{s \alpha} h f_{s y}
\end{array} \rightarrow|A|=\rho_{s \alpha} h f_{s y}+|B| \geqslant|B|\right.\right.
$$
(B-7).

Thus, $|B| \leqslant \rho_{s \alpha} h f_{s y}$ is a sufficient condition to have $|A| \geqslant|B|$, and hence to have a nonnegative dissipation for all $A$ satisfying

Inserting (B-3) in the condition $|B| \leqslant \rho_{s \alpha} h f_{s y}$ :

$$
\left|s_{r, \alpha}\left(\boldsymbol{K}^{s} \cdot\left(\boldsymbol{M}^{v w} \cdot \underline{w}-\underline{v}^{p}\right)\right) \cdot \underline{e}_{\alpha}\right| \leqslant \rho_{s \alpha} h f_{s y}
$$

and dividing by $s_{r, \alpha}$ :

$$
\left|\left(\boldsymbol{K}^{s} \cdot\left(\boldsymbol{M}^{v w} \cdot \underline{w}-\underline{v}^{p}\right)\right) \cdot \underline{e}_{\alpha}\right| \leqslant \frac{\rho_{s \alpha} h}{s_{r, \alpha}} f_{s y}
$$

it can be identified:

$$
\left|\left(\boldsymbol{K}^{s} \cdot\left(\boldsymbol{M}^{v w} \cdot \underline{w}-\underline{v}^{p}\right)\right) \cdot \underline{e}_{\alpha}\right|=\left|\left(\underline{w} \cdot \boldsymbol{G}-\boldsymbol{E} \cdot \underline{v}^{p}\right) \cdot \underline{e}_{\alpha}\right|=\left|\underline{q}_{v} \cdot \underline{e}_{\alpha}\right|=\left|q_{v, \alpha}\right| \leqslant \frac{\rho_{s \alpha} h}{s_{r, \alpha}} f_{s y}
$$

which has to hold for all $\underline{q}_{v}$ satisfying (5-24):

$$
\left\{\begin{array}{l}
\left|q_{v, \alpha}\right| \leqslant \frac{\rho_{s \gamma} h}{s_{r, \alpha}} f_{s y} \\
\forall q_{v, \alpha} \backslash\left|q_{v, \alpha}\right| \leqslant \frac{\rho_{c} h}{s_{r, \alpha}} k_{t} f_{c t}
\end{array} \rightarrow \frac{\rho_{c} h}{s_{r, \alpha}} k_{t} f_{c t} \leqslant \frac{\rho_{s \alpha} h}{s_{r, \alpha}} f_{s y}\right.
$$

Finally, this condition can be expressed as a limitation of the average tension stiffening concrete stress coefficient:

$$
\frac{\rho_{c} h}{s_{r, \alpha}} k_{t} f_{c t} \leqslant \frac{\rho_{s \alpha} h}{s_{r, \alpha}} f_{s y} \leftrightarrow k_{t} \leqslant \frac{\rho_{s \alpha}}{\rho_{c}} \frac{f_{s y}}{f_{c t}}
$$

\section{Appendix C}

This appendix is devoted to demonstrate that the concrete stresses at cracks (4-28) and the macro concrete stress at cracks (5-8) are directly related by the identity $\underline{g}=\left(g_{n}, g_{t}\right)=\underline{q}_{r} \frac{s_{r}}{\rho_{c} h}$, as stated in (5-13).

With the definition of $\mathbb{M}^{\varepsilon w}$ given in Section 4.4, concrete stress at cracks can be expressed as:

$$
\underline{g}=\left[\mathbb{C}_{c}(d):\left(\boldsymbol{\epsilon}-\boldsymbol{\varepsilon}^{r}\right)-\boldsymbol{\sigma}^{\tau}\right]_{n n, t n}=s_{r}{ }^{t} \mathbb{M}^{\varepsilon w}:\left(\mathbb{C}_{c}(d):\left(\boldsymbol{\epsilon}-\mathbb{M}^{\varepsilon w} \cdot \underline{w}\right)-\boldsymbol{\sigma}^{\tau}\right)
$$

The term of the previous equation depending on the tension stiffening can be expressed as functions of the internal variables:

$$
s_{r}{ }^{t} \mathbb{M}^{\varepsilon w}: \boldsymbol{\sigma}^{\tau}={ }^{t} \boldsymbol{M}^{v w} \cdot \boldsymbol{K}^{s} \cdot\left(\boldsymbol{M}^{v w} \cdot \underline{w}-\underline{v}^{p}\right) S_{r} /\left(\rho_{c} h\right)
$$

The demonstration of (C-2) is given hereinafter.

On the one hand, the product $s_{r}{ }^{t} \mathbb{M}^{\varepsilon w}: \boldsymbol{\sigma}^{\tau}$ accounts for the $n n$ and $t n$ components of the average tension stiffening stress in concrete $\boldsymbol{\sigma}^{\tau}$

$$
\left.s_{r}{ }^{t} \mathbb{M}^{\varepsilon w}: \boldsymbol{\sigma}^{\tau}=\begin{array}{c}
\boldsymbol{\sigma}_{x x}^{\tau} \sin ^{2} \theta_{r}+\boldsymbol{\sigma}_{y y}^{\tau} \cos ^{2} \theta_{r} \\
\left(\boldsymbol{\sigma}_{y y}^{\tau}-\boldsymbol{\sigma}_{x x}^{\tau}\right) \sin \theta_{r} \cos \theta_{r}
\end{array}\right)
$$

On the other hand, the product $\boldsymbol{K}^{s} \cdot\left(\boldsymbol{M}^{v w} \cdot \underline{w}-\underline{v}^{p}\right) \boldsymbol{S}_{r} /\left(\rho_{c} h\right)$ is analyzed taking into account that $\boldsymbol{K}^{s}$ is a diagonal tensor (53) and that $\boldsymbol{M}^{v w} \cdot \underline{w}=\underline{v}$ :

$$
\begin{aligned}
& \frac{K_{x x}^{s} s_{r}}{\rho_{c} h}\left(v_{x}-v_{x}^{p}\right)=\frac{2 s_{r, x} \rho_{s x}}{\rho_{c} \phi_{x}}\left|K_{x x}^{\tau}\right| \frac{\rho_{c} h}{s_{r, x}} \frac{s_{r}}{\rho_{c} h}\left(v_{x}-v_{x}^{p}\right)=\frac{2 s_{r, x} \rho_{s x}}{\rho_{c} \phi_{x}}\left|K_{x x}^{\tau}\right|\left(v_{x}-v_{x}^{p}\right)\left|\sin \theta_{r}\right|=\sigma_{x x}^{\tau}\left|\sin \theta_{r}\right| \\
& \frac{K_{y y}^{s} s_{r}}{\rho_{c} h}\left(v_{y}-v_{y}^{p}\right)=\frac{2 s_{r, y} \rho_{s y}}{\rho_{c} \phi_{y}}\left|K_{y y}^{\tau}\right| \frac{\rho_{c} h}{s_{r, y}} \frac{s_{r}}{\rho_{c} h}\left(v_{y}-v_{y}^{p}\right)=\frac{2 s_{r, y} \rho_{s y}}{\rho_{c} \phi_{y}}\left|K_{y y}^{\tau}\right|\left(v_{y}-v_{y}^{p}\right) \cos \theta_{r}=\sigma_{y y}^{\tau} \cos \theta_{r}
\end{aligned}
$$


with the definition (4-29) of the average tension stiffening stress in concrete $\boldsymbol{\sigma}^{\tau}$. When multiplying this result by the transformation matrix ${ }^{t} \boldsymbol{M}^{v w}$ (3-6):

$$
\left.\left.{ }^{t} \boldsymbol{M}^{v w} \cdot \boldsymbol{K}^{s} \cdot\left(\boldsymbol{M}^{v w} \cdot \underline{w}-\underline{v}^{p}\right) \frac{s_{r}}{\rho_{c} h}=\left(\begin{array}{cc}
\left|\sin \theta_{r}\right| & \cos \theta_{r} \\
-\cos \theta_{r} \operatorname{sign}\left(\theta_{r}\right) & \sin \theta_{r}
\end{array}\right) \cdot \begin{array}{c}
\sigma_{x x}^{\tau}\left|\sin \theta_{r}\right| \\
\sigma_{y y}^{\tau} \cos \theta_{r}
\end{array}\right)=\begin{array}{c}
\sigma_{x x}^{\tau} \sin ^{2} \theta_{r}+\sigma_{y y}^{\tau} \cos ^{2} \theta_{r} \\
\left(\sigma_{y y}^{\tau}-\sigma_{x x}^{\tau}\right) \sin \theta_{r} \cos \theta_{r}
\end{array}\right)
$$

the same expression as in (C-4) is found, and (C-2) is demonstrated.

Finally, substituting (C-2) in (C-1) and rearranging the terms:

$$
\begin{aligned}
\underline{g} & =s_{r}{ }^{t} \mathbb{M}^{\varepsilon w}: \mathbb{C}_{c}(d):\left(\boldsymbol{\epsilon}-\mathbb{M}^{\varepsilon w} \cdot \underline{w}\right)-{ }^{t} \boldsymbol{M}^{v w} \cdot \boldsymbol{K}^{s} \cdot \frac{\left(\boldsymbol{M}^{v w} \cdot \underline{w}-\underline{v}^{p}\right) s_{r}}{\left(\rho_{c} h\right)} \\
& =s_{r} \mathbb{C}_{c}(d): \mathbb{M}^{\varepsilon w} \boldsymbol{\epsilon}-s_{r}\left({ }^{t} \mathbb{M}^{\varepsilon w}: \mathbb{C}_{c}(d): \mathbb{M}^{\varepsilon w}+{ }^{t} \boldsymbol{M}^{v w} \cdot \boldsymbol{K}^{s} \cdot \frac{\boldsymbol{M}^{v w}}{\left(\rho_{c} h\right)}\right) \cdot \underline{w}+{ }^{t} \boldsymbol{M}^{v w} \cdot \frac{\boldsymbol{K}^{s} \underline{v}^{p} s_{r}}{\left(\rho_{c} h\right)} \\
& =\left(\boldsymbol{\epsilon}: \mathbb{B}(d)-\boldsymbol{D}(d) \cdot \underline{w}+\boldsymbol{G}(d) \cdot \underline{v}^{p}\right) s_{r} /\left(\rho_{c} h\right)=\underline{q}_{r} s_{r} /\left(\rho_{c} h\right)
\end{aligned}
$$

the relationship between $\underline{g}$ and $\underline{q}_{r}$ is found.

\section{Appendix D}

This appendix is devoted to the estimation of the parameters which, even if their physical signification is clear, classical tests do not give enough information to determine them. Some indications about the determination of these parameters are given below:

- In absence of experimental tests values, the fracture energy $G_{f}$ can be estimated by the expression given by MC10:

$$
G_{f}=73 f_{c}^{0.18}
$$

where concrete compressive strength $f_{c}$ is expressed in MPa and fracture energy in $G_{f}$ in $\mathrm{J} / \mathrm{m}^{2}$.

- Parameters $\alpha_{u}$ and $\alpha_{r}$, which define concrete bridging stress cyclic behavior, may depend on concrete characteristics, especially on the aggregate size and form. If no tests are carried out to identify them, it is recommended to use an unloading slope ratio $\alpha_{u} \in[0.01,0.2]$ and crack reclosing ratio $\alpha_{r} \in[0.01,0.1]$. It is remarked that when the applied loading creates significant tangential crack displacements $w_{t}$, the crack reclosing ratio should be relatively high, in order to reproduce the dilatancy effect, which is not directly considered in the model formulation.

- Aggregate interlock parameters $T_{o}$ and $T_{1}$ may depend on the aggregate characteristics and the type and magnitude of the loading. Recommended values are $T_{o}=\in[0.01,0.1] \mathrm{MPa}$ and $T_{1} \in[1,20] \mathrm{GPa} / \mathrm{m}$, where the lower bounds of the ranges are related to expected high values of crack opening $w_{n}$ (or low values of normal concrete stress at cracks) and to cyclic loadings.

- The theoretical average crack spacing of the equivalent tie beams in the $x$ and $y$ directions can be estimated with civil engineering codes formulas for maximum crack spacing, after the transformation to average spacing values by dividing them by 1.7 (for the case of MC10 and EC2). However, it is recommended to use the following optimized formula for average crack spacing given by [14]:

$$
s_{r \alpha}=1.37 c_{\alpha}+0.116 \phi_{\alpha} / \rho_{s \alpha}
$$

where $c_{\alpha}$ is the concrete cover of steel reinforcement bars in the $\alpha$ direction. Finally, the experimental values for the average crack spacing should be used when available.

- The energy release rate threshold $k_{o}$ is calculated from the concrete stress at the damage onset $\sigma_{d}$ with:

$$
k_{0}=\frac{\left(1-\gamma_{d}\right) \sigma_{d}^{2}}{2 E_{c}}
$$

where it is recommended to use $\gamma_{d} \in[0.2,0.3]$ for SLS and $\sigma_{d} \in\left[f_{c} / 4, f_{c} / 2\right]$.

- The local bond-slip tangent stiffness can be estimated to $K_{l} \in\left[10^{10}, 10^{11}\right] \mathrm{Pa} / \mathrm{m}$, depending on the characteristic values of the obtained steel-concrete slip since it should correspond to the secant stiffness of realistic bond-slip laws as the given by MC10.

- The retained tension stiffening coefficient may vary between $k_{t} \in[0.1,0.6]$ :

o $\quad k_{t}=0.6$ when calculating crack openings with the same assumptions as MC10 and EC2.

o $k_{t} \approx 1 / 3$ when the computation is done for representing the monotonic mechanical behavior.

o $k_{t} \in[0.1,0.2]$ when time dependent effects in concrete are important, or in cyclic loadings implying bond degradation. 


\section{References}

[1] Belletti B, Cerioni R, Iori I. Physical Approach for Reinforced-Concrete (PARC) membrane elements. J Struct Eng 2001;127:1412-26.

[2] Benmansour MB Modélisation du comportement cyclique alterné du béton armé. Application à divers essais statiques de poteaux [PhD Thesis]. Ecole Nationale des Ponts et Chaussées; 1997.

[3] CEN. EN 1992-1-1. Eurocode 2: design of concrete structures: Part 1-1; 2005.

[4] Ciarlet PG. A justification of a nonlinear model in plate theory. Comput Method Appl M 1979;17-18(Part 1):227-58.

[5] Combescure C, Dumontet H, Voldoire F. Homogenized constitutive model coupling damage and debonding for reinforced concrete structures under cyclic solicitations. Int J Solids Struct 2013;50(24):3861-74.

[6] Combescure C, Dumontet H, Voldoire F. Dissipative Homogenised Reinforced Concrete (DHRC) constitutive model dedicated to reinforced concrete plates under seismic loading. Int J Solids Struct 2015;73-74:78-98.

[7] Destuynder P, Theodory Ch. Homogénéisation de structures minces en béton armé. RAIRO-Math Model Num 1986;20(1):47-74.

[8] EDF. Code_Aster, general public licensed structural mechanics finite element software; 2017. Internet site <http://www.code-aster.org>.

[9] Erlicher S, Point P. Endochronic theory, non-linear kinematic hardening rule and generalized plasticity: a new interpretation based on generalized normality assumption. Int J Solids Struct 2006;43:4175-200.

[10] Erlicher S, Point P. Pseudopotentials and loading surfaces for an endochronic plasticity theory with isotropic damage. J Eng Mech - ASCE 2008;134 (10):832-42.

[11] Farra B, Jaccoud JP. Influence du Béton et de l'armature sur la fissuration des structures en Béton. Rapport des essais de tirants sous déformation imposée de courte durée. Département de Génie Civil, Ecole Polytechnique Fédérale de Lausanne, Publication No. $140 ; 1993$.

[12] Fédération Internationale du Béton fib. Model Code 2010. Final draft; 2011.

[13] Halphen B, Nguyen QS. Sur les matériaux standards généralisés. J Méc 1975;14:39-63.

[14] Huguet M. Homogenised stress resultant constitutive model for cracking in RC plates under seismic loadings. [PhD Thesis]. Ecole Centrale Nantes/EGIS Industries; 2016.

[15] Hsu TTC, Zhu RRH. Softened membrane model for reinforced concrete elements in shear. ACI Struct J 2002;99(4):460-9.

[16] Kaufmann W. Strength and deformation of structural concrete subjected to in-plane shear and normal forces. [PhD Thesis]. Institute of Structural Engineering, Swiss Federal Institute of Technology Zurich; 1998.

[17] Kaufmann W, Marti P. Structural concrete: cracked membrane model. J Struct Eng 1998;98(4):287-98.

[18] Lemaitre J, Chaboche JL. Mechanics of solid materials. Cambridge University Press; 1994.

[19] Li B, Maekawa K, Okamura H. Contact density model for stress transfer across cracks in concrete. J Fac Eng U Tokyo 1989;40:9-52.

[20] Markovic D, Koechlin P, Voldoire F. Reinforced concrete structures under extreme loading: stress resultant Global Reinforced Concrete Models (GLRC). In: Proc of COMPDYN 2007, Rethymno, Greece; 2007.

[21] Marti P, Alvarez M, Kaufmann W, Sigrist V. Tension Chord Model for structural concrete. SEI 1998;4(98):287-98. IABSE, Zurich, Switzerland.

[22] Pimentel M, Brühwiler E, Figueiras J. Extended cracked membrane model for the analysis of RC panels. Eng Struct 2010;32:1964-75.

[23] Point N, Erlicher S. Convex analysis and thermodynamics. Kinetic Relat Models - AIMS 2013;6(4):945-54.

[24] Richard B, Ragueneau F. Continuum damage mechanics based model for quasi brittle materials subjected to cyclic loadings: formulation, numerical implementation and applications. Eng Fract Mech 2013;98:383-406.

[25] Rivillon P, Gabs A. Technical Report “Rapport NEEM 09 26023877-C”. CSTB; 2011.

[26] Rossi P, Wu X, Le Maou F, Belloc A. Scale effect on concrete in tension. Mater Struct 1994;27:437-44.

[27] Ruocci G, Rospars C, Bisch P, Erlicher S, Moreau G. Cracks distance and width in reinforced concrete membranes: experimental results from cyclic loading histories. In: Proceedings of the 15th World Conference on Earthquake Engineering, Lisbon, Portugal; 2012.

[28] Suquet P. Overall potentials and extremal surfaces of power law or ideally plastic composites. J Mech Phys Solids 1993;41:981-1002.

[29] Vecchio FJ. Disturbed stress field model for reinforced concrete: formulation. J Struct Eng 2000;126(9):1070-7.

[30] Vecchio FJ, Collins MP. The modified compression-field theory for reinforced concrete elements subjected to shear. ACI J 1986;83(2):219-31.

[31] Walraven J. Fundamental analysis of Aggregate Interlock. J Struct Div 1981;107(11):2245-70.

[32] Zanuy C, De la Fuente P, Albajar L. Estimation of parameters defining negative tension stiffening. Eng Struct 2010;32(10):3355-62. 